Gérard Ben Arous · Jiří Černý · Thomas Mountford

\title{
Aging in two-dimensional Bouchaud's model
}

Received: 20 November 2003 / Revised version: 26 October 2004 /

Published online: 10 February 2005 - (C) Springer-Verlag 2005

Abstract. Let $E_{x}$ be a collection of i.i.d. exponential random variables. Symmetric Bouchaud's model on $\mathbb{Z}^{2}$ is a Markov chain $X(t)$ whose transition rates are given by $w_{x y}=v \exp \left(-\beta E_{x}\right)$ if $x, y$ are neighbours in $\mathbb{Z}^{2}$. We study the behaviour of two correlation functions: $\mathbb{P}\left[X\left(t_{w}+t\right)=X\left(t_{w}\right)\right]$ and $\mathbb{P}\left[X\left(t^{\prime}\right)=X\left(t_{w}\right) \forall t^{\prime} \in\left[t_{w}, t_{w}+t\right]\right]$. We prove the (sub)aging behaviour of these functions when $\beta>1$.

\section{Introduction}

We explore in this paper a mechanism for aging of Markovian dynamics in complex random media proposed by J.P. Bouchaud. This mechanism is based on trapping. More precisely if a Markov process moves in a very complex landscape of energy, it should spend most of its time in the deep valleys of this landscape; its long time behaviour should be essentially ruled by three features: the (short) transits between these valleys, the relative positions of these valleys, and the (long) exit times from these valleys (which are usually exponentially distributed with parameters proportional to the (random) depth of the valley). Aging (see [Ben02] for a recent survey) would then simply be the consequence of the following mechanism: the older the system is the more space it has explored, the deeper valley it is stuck in. So that the process essentially stays put for longer and longer periods of time.

In order to capture the core of this appealing picture, Bouchaud proposed a very simple effective model of trapping on a graph. Let us describe it here. Let $G=(\mathcal{V}, \mathcal{E})$ be a connected graph. The vertices of $G$ should be seen as valleys and the graph structure as the description of the communication between these valleys. The random landscape is now given by a collection of i.i.d. random variables $\boldsymbol{E}=\left\{E_{x}\right\}_{x \in \mathcal{V}}$, exponentially distributed with mean $1 . E_{x}$ should be seen as the depth of the valley at $x$. We consider a random "Gibbs" measure $\tau$ on $\mathcal{V}$ with mass

G. Ben Arous: École Polytechnique Fédérale de Lausanne, 1015 Lausanne, Switzerland

G. Ben Arous: Courant Institute of Mathematical Sciences, 251 Mercer Street, New York,

N.Y. 10012-1185, USA. e-mail: benarous@cims . nyu . edu

J. Černý: Weierstrass Institute for Applied Analysis and Stochastics (WIAS),

Mohrenstr. 39, 10117 Berlin, Germany. e-mail: cerny@wias-berlin. de

T. Mountford: Département de Mathématiques, École Polytechnique Fédérale de Lausanne, 1015 Lausanne, Switzerland. e-mail: thomas . mount ford@epfl . ch

Mathematics Subject Classification (2000): 82D30, 82C41, 60F17

Key words or phrases: Aging - Trap model - Lévy process - Random walk - Time change 
of vertex $x$ given by

$$
\tau_{x}=e^{\beta E_{x}},
$$

where $\beta>0$ is the inverse temperature. We define Bouchaud's trap model as a continuous time Markov chain $X(t)$ on $\mathcal{V}$ by the jump rates

$$
w_{x y}=v \exp \left[-\beta\left((1-a) E_{x}-a E_{y}\right)\right] \quad \text { if }(x, y) \in \mathcal{E},
$$

and zero otherwise. The constant $v$ fixes only the time scale and will be fixed later, and $a \in[0,1]$ tunes the influence of neighbouring valleys on jumping rates. The simplest case is $a=0$, then it is clear that the Markov chain $X$ is a random time change of the simple random walk on the graph. Notice that the random measure $\tau$ is reversible for all values of $a$ and $\beta$.

This model has been introduced in the physics literature (see [Bou92, MB96, BM97]) on a large complete graph initially as an ansatz for the dynamics of the Random Energy Model (see [BBG03a, BBG03b] for a rigorous study). It was then considered on the graph $\mathbb{Z}^{d}$ in [RMB00] and first time rigorously studied by [FIN02] for $d=1$ and $a=0$, then by [BČ04] for $d=1$ and general $a$. We study here Bouchaud's trap model on the lattice $\mathbb{Z}^{2}$ in $a=0$ case. This case has been sketched in [RMB00] with a concept of partial equilibrium which is rather difficult to justify. For convenience we will choose $v=1 /(2 d)=1 / 4$. The process that we consider is started at the origin, $X(0)=0$.

Let us state our aging result. We consider the following two-point function:

$$
R\left(t_{w}, t_{w}+t\right)=\mathbb{P}\left[X\left(t_{w}+t\right)=X\left(t_{w}\right) \mid \boldsymbol{E}\right],
$$

which is the probability that the process is at the same site at time $t_{w}+t$ as it was at time $t_{w}$. We prove the aging behaviour for the function $R$.

Theorem 1.1. For all $\beta>1$ and $a=0$ there exists a function $R(\theta)$ such that for $\mathbb{P}$-a.e. realisation of the environment $\boldsymbol{E}$

$$
\lim _{t_{w} \rightarrow \infty} R\left(t_{w}, t_{w}+\theta t_{w}\right)=R(\theta)
$$

Moreover, the function $R(\theta)$ can be explicitly calculated (see Proposition 7.1) and it satisfies

$$
\lim _{\theta \rightarrow 0} R(\theta)=1 \text { and } \lim _{\theta \rightarrow \infty} R(\theta)=0 .
$$

We further study the following two-point function:

$$
\Pi\left(t_{w}, t_{w}+t\right)=\mathbb{P}\left[X\left(t^{\prime}\right)=X\left(t_{w}\right) \forall t^{\prime} \in\left[t_{w}, t_{w}+t\right] \mid \boldsymbol{E}\right],
$$

which is the probability that the process does not jump between the times $t_{w}$ and $t_{w}+t$. We show sub-aging behaviour for this two-point function. 
Theorem 1.2. For all $\beta>1$ and $a=0$ there exists a function $\Pi(\theta)$ such that for $\mathbb{P}$-a.e. realisation of the environment $\boldsymbol{E}$

$$
\lim _{t_{w} \rightarrow \infty} \Pi\left(t_{w}, t_{w}+\theta \frac{t_{w}}{\log t_{w}}\right)=\Pi(\theta) .
$$

The function $\Pi(\theta)$ can be again made explicit (see Proposition 8.2) and it satisfies the same relations (5) as $R(\theta)$.

Remarks. 1. Our results stay valid if we replace the condition that $E_{x}$ is exponentially distributed with mean 1 by the weaker condition on the mean waiting time

$$
\mathbb{P}\left[\tau_{x}>0\right]=1 \quad \text { and } \quad \lim _{u \rightarrow \infty} u^{\alpha} \mathbb{P}\left(\tau_{0} \geq u\right)=K \quad \text { with } \alpha \in(0,1),
$$

and $K \in(0, \infty)$. This condition is easy to verify for the original distribution with $\alpha=1 / \beta, K=1$. For the rest of the paper the condition (8) is in force, we also assume for simplicity that $K=1$. The limiting functions $R(\theta)$ and $\Pi(\theta)$ do not depend on the choice of distribution of $\tau_{x}$ verifying these conditions.

2. Unlike as in $d=1$ case [FIN02, BČ04] we study here the so called quenched two-point functions, that means that we obtain (sub)aging for a.e. environment. The averaged results are an easy consequence of our theorems. To complete the picture, we recall the results of [FIN02, BČ04]. It was proved that the averaged two-point functions satisfy

$$
\begin{aligned}
& \lim _{t_{w} \rightarrow \infty} \mathbb{E} R\left(t_{w}, t_{w}+\theta t_{w}\right)=R_{1}(\theta) \\
& \lim _{t_{w} \rightarrow \infty} \mathbb{E} \Pi\left(t_{w}, t_{w}+\theta t_{w}^{(1-a) /(1+\alpha)}\right)=\Pi_{1, a}(\theta) .
\end{aligned}
$$

Note also that in $d=1$ the analogous quenched results are not valid.

3. The $d \geq 3, a=0$ case is treated in [Čer03]. Very similar results are obtained there. Namely, it is proved that

$$
\begin{aligned}
\lim _{t_{w} \rightarrow \infty} R\left(t_{w}, t_{w}+\theta t_{w}\right) & =R(\theta), \\
\lim _{t_{w} \rightarrow \infty} \Pi\left(t_{w}, t_{w}+\theta t_{w}\right) & =\Pi_{d}(\theta),
\end{aligned}
$$

with the same function $R(\theta)$ as in Theorem 1.1. The change of the scale for the two-point function $\Pi$ reflects the fact that the simple random walk in $d \geq 3$ visits any site only finitely many times. As the methods used in our paper do not use substantially the fact that $d=2$, the proof for $d \geq 3$ is, except for some problems of technical character, very similar to the one presented here. The major difference comes from the fact that the computations should be adjusted to the different formulae for Green's function and hitting probabilities.

4. The case $d=2, a>0$ is much harder and will be treated elsewhere. In this case Bouchaud's trap model is not longer a time change of the simple random walk but some type of reversible random walk in random environment. 
5. The choice $v=1 / 4$ assures that the mean waiting time of $X$ at site $x$ is equal to $\tau_{x}$. Therefore, the process $X(t)$ stays at the site $x$ an exponentially distributed time with mean $\tau_{x}$ and then it jumps with equal probability to one of the four neighbouring sites. Formally, let $X_{d}(i), i=0,1, \ldots$, denote a discrete time simple random walk on $\mathbb{Z}^{2}$ started at the origin, and let $e_{i}$ be a collection of i.i.d. exponential random variables with mean one. We use $S(n)$ to denote the "time change" of the simple random walk

$$
S(n)=\sum_{i=0}^{n-1} e_{i} \tau_{X_{d}(i)}
$$

Then $X(t)=X_{d}(j)$ if $S(j) \leq t<S(j+1)$. Since the random variables $\tau_{x}$ and $E_{x}$ are directly related by (1) we abuse terminology slightly, and call $\tau_{x}$ also depth of the trap at $x$. Actually, we do not use $E_{x}$ later in this paper and the word "depth" refers always to $\tau_{x}$.

6. Our results would probably remain valid if the time change of a finite range, symmetric random walk were used to define the process $X$. As such modification would only bring difficulties into the already quite technical reasoning, we prefer to stick to the simple definition.

The results of both theorems can be described heuristically in the following way. After time $t_{w}$ the system is typically in a trap whose mean waiting time is of order $t_{w} / \log t_{w}$ (as can be seen from Theorem 1.2). After passing a time of that order in the trap the process $X$ makes excursions from it and returns there of order $\log t_{w}$ times before time $(1+\theta) t_{w}$. The time that these excursions take is typically much smaller than the time that $X$ spends in the deep trap. Then $X$ leaves the neighbourhood of this trap and continues to explore the lattice.

We describe here the strategy that will be used to prove both the theorems. Let $n \in \mathbb{N}$. We consider the process $X(t)$ only before the exit from the disk $\mathbb{D}(n)$ with the area $m 2^{n} n^{1-\alpha}$ around the origin. The constant $m$ will be chosen later in order that the walk can stay a sufficiently long time inside $\mathbb{D}(n)$. We are interested mainly in the time that the walk spends in traps that are deeper than $\varepsilon 2^{n / \alpha} / n$, for $\varepsilon \ll 1$ to be fixed later (such traps will be referred to as deep traps). In the disk $\mathbb{D}(n)$ there are approximately $m n / \varepsilon^{\alpha}$ such traps. Since the probability of hitting a particular point in $\mathbb{D}(n)$, that is sufficiently far from the walk initial point, before the exit from $\mathbb{D}(n)$ is of order $n^{-1}$, the walk has a reasonable chance to hit at least one deep trap. The constant $\varepsilon$ will be chosen small enough to ensure that the walk spends a negligible proportion of time in shallower traps.

We cut the trajectory of the process $X$ into short parts. Every part is finished when $X$ exits for the first time the disk of area $2^{n} n^{\gamma}$ around the initial point of the part. At this moment a new part is started. Clearly, we should take $\gamma<1-\alpha$. For every such part we look at the time that the walk spends in the traps which we have specified in the previous paragraph. It will be proved that, with overwhelming probability, the walk hits at most one such trap in every part. Moreover, the same trap is almost never hit again in the next parts before the exit from $\mathbb{D}(n)$. To the $i$-th part of the trajectory we associate a random variable $s_{i}$ that we call score of that 
part, and that is roughly the time spent by $X$ in the deep trap that was hit during this part (the score will be defined in Section 2). It will be proved that for $n$ sufficiently large the random variables $s_{i}$ are essentially independent and the properly rescaled trajectory of the sum $\sum s_{i}$ converges to a pure jump, increasing Lévy process. It will be also shown that this sum is a good approximation for the properly rescaled time change $S(n)$.

The proof of both theorems relies on the fact that the events that we are interested in, that is the probabilities of staying a long time at the same place, mainly occur if properly rescaled values of times $t_{w}$ and $t_{w}+t$ falls into one jump of the Lévy process from the previous paragraph, or more precisely if the intersection of the range of the Lévy process with the rescaled interval $\left[t_{w}, t_{w}+t\right]$ is empty. The probability of such an event is easy to calculate using arcsine law for Lévy processes (see [Ber96]).

The theorems are proved in Sections 7 and 8 where the reader can also find the explicit expressions for the functions $R(\theta)$ and $\Pi(\theta)$. The proof of the convergence of properly rescaled sums of scores occupies Sections 2-6.

Throughout the paper we will deal with typically non integer objects such as $\sqrt{n 2^{n}}$ or $2^{n} n^{\gamma}$ for discrete valued processes. In these contexts it is to be understood that the quantity referred to is the integer part.

\section{The coarse-graining of $X(t)$}

We introduce some notations needed later. We use $D_{x}(m)$, and $B_{x}(m)$ to denote the disk, resp. the box, with area $m$ around the site $x$. If $x$ is omitted the disk (box) is centred around the origin. Both these objects are understood as subsets of $\mathbb{Z}^{2}$. In the following we will very often use the claim that the disk $D(m)$ contains $m$ sites from $\mathbb{Z}^{2}$, although it is not precisely true. Precisely $D(m)$ will be the disk of radius $r$, where $r$ is the infimum of the radii of disks centred at the origin containing at least $m$ lattice points. Any error we introduce by this consideration will be negligible for $m$ large enough.

Let $n \in \mathbb{N}$ large. We consider the process $X(t)$ before the first exit from the disk $\mathbb{D}(n) \equiv D\left(m 2^{n} n^{1-\alpha}\right)$. We write

$$
\begin{gathered}
\Lambda_{d}(n)=\inf \left\{i \in \mathbb{N}: X_{d}(i) \notin \mathbb{D}(n)\right\}, \\
\Lambda(n)=\inf \{t \in \mathbb{R}: X(t) \notin \mathbb{D}(n)\}
\end{gathered}
$$

for the exit times of the discrete, resp. continuous, time process from $\mathbb{D}(n)$. We will often skip the dependence on $n$ in our notation.

We use $T_{\varepsilon}^{M}(n)$ to denote the set

$$
T_{\varepsilon}^{M}(n)=\left\{x \in \mathbb{D}(n): \frac{\varepsilon 2^{n / \alpha}}{n} \leq \tau_{x}<\frac{M 2^{n / \alpha}}{n}\right\} .
$$

If $M$ or $\varepsilon$ are omitted, it is understood $M=\infty$, resp. $\varepsilon=0$. The constants $\varepsilon$ and $M$ will be chosen later. However, we always suppose that $\varepsilon \ll 1 \ll M$. We call the traps from $T^{\varepsilon}$ shallow traps, $T_{\varepsilon}^{M}$ is the set of deep traps, and $T_{M}$ is the set of very deep traps. We will show that as $M$ becomes large the probability of hitting 
a point in $T_{M}$ before time of order $2^{n / \alpha}$ (which is the time that $X$ typically spends in $\mathbb{D}(n)$ ) will be negligible, while as $\varepsilon$ becomes small the amount of time spent by process $X$ before time of order $2^{n / \alpha}$ in sites of $T^{\varepsilon}$ will be very small.

We write $\mathcal{E}(n)$ for the set of sites that are sufficiently far from the set $T_{\varepsilon}^{M}(n)$,

$$
\mathcal{E}(n)=\mathbb{D}(n) \backslash \bigcup_{y \in T_{\varepsilon}^{M}(n)} D_{y}\left(2^{n} n^{-\kappa}\right) .
$$

The constant $\kappa=\kappa(\alpha)$ can be taken arbitrarily large, but will be fixed while $n \rightarrow \infty$. The value $\kappa=5 /(1-\alpha)$ is sufficient for our purposes. The role of the set $\mathcal{E}(n)$ will be clarified later.

Further, we introduce a function $L(u)$ satisfying

$$
\mathbb{P}\left[\tau_{0} \geq u\right]=u^{-\alpha} L(u)
$$

From (8) we know that $\lim _{u \rightarrow \infty} L(u)=1$. It is also not difficult to see that $L$ is bounded.

We write $\chi(A)$ for the indicator function of the set $A$. We use the letters $C$, $c$ to denote positive constants that have no particular importance. The value of these constants can change during computations. On the other hand, the letter $K$ is reserved for constants with particular meaning.

We define now the coarse-graining of the trajectory of the process $X$. Let $\gamma<$ $1-\alpha$. We set $j_{0}^{n}=0$, and then we define recursively

$$
j_{i}^{n}=\min \left\{k>j_{i-1}^{n}: X_{d}(k) \notin D_{X_{d}\left(j_{i-1}^{n}\right)}\left(2^{n} n^{\gamma}\right)\right\},
$$

with the convention that the minimum of an empty set is equal to infinity. We use $x_{i}^{n}$ to denote the starting points of the parts of the trajectory, $x_{i}^{n}=X_{d}\left(j_{i}^{n}\right)$. The range of $X_{d}$ between the times $j$ and $k$ is denoted by $X_{d}[j, k)$, i.e. $X_{d}[j, k)=\left\{X_{d}(l)\right.$ : $j \leq l<k\}$.

We will now define the score $s_{i}^{n}$ of the part $X_{d}\left[j_{i}^{n}, j_{i+1}^{n}\right)$. Let $\lambda_{1}$ be the first time when $X_{d}$ hits a deep trap after the start of this part,

$$
\lambda_{1}=\min \left\{k \geq j_{i}^{n}: X_{d}(k) \in T_{\varepsilon}^{M}\right\} .
$$

Let $y=X_{d}\left(\lambda_{1}\right)$ be the first visited deep trap after time $j_{i}^{n}$. Further, let $\lambda_{2}$ be the exit time from the disk $D_{y}\left(2^{n} n^{-\kappa}\right)$,

$$
\lambda_{2}=\min \left\{k>\lambda_{1}: X_{d}(k) \notin D_{y}\left(2^{n} n^{-\kappa}\right)\right\} .
$$

The last time that we need is

$$
\lambda_{3}=\min \left(\left\{k>\lambda_{1}: X_{d}(k) \in T_{\varepsilon}^{M} \backslash\{y\}\right\} \cup\left\{k \geq \lambda_{2}: X_{d}(k) \in T_{\varepsilon}^{M}\right\}\right) .
$$

It is the first time after $\lambda_{1}$ when $X_{d}$ hits a deep trap, but we do not consider the successive hits of the trap $y$ before the time $\lambda_{2}$, so it is possible that $X_{d}\left(\lambda_{3}\right)=y$. 
If $\lambda_{1}<\lambda_{2} \leq j_{i+1}^{n} \leq \lambda_{3}, j_{i+1}^{n} \leq \Lambda_{d}$, and $y$ is farther than $\sqrt{\pi^{-1} 2^{n} n^{-\kappa}}$ from the border of $D_{x_{i}^{n}}\left(2^{n} n^{\gamma}\right)$, we define the score associated with interval $\left[j_{i}^{n}, j_{i+1}^{n}\right)$ by

$$
s_{i}^{n}=\sum_{k=\lambda_{1}}^{\lambda_{2}} e_{k} \tau_{y} \chi\left(X_{d}(k)=y\right) .
$$

The last condition assures that the movement of $X$ inside $D_{y}\left(2^{n} n^{-\kappa}\right)$ is not influenced by the border of $D_{x_{i}^{n}}\left(2^{n} n^{\gamma}\right)$. If $\lambda_{1} \geq j_{i+1}^{n}$ and $j_{i+1}^{n} \leq \Lambda_{d}$, we set $s_{i}^{n}=0$. In both previous cases the score is simply the time spent in the first visited deep trap. In all other cases we set $s_{i}^{n}=\infty$. This value has no particular meaning, it only marks the parts of the trajectory where something "unusual" happens. By unusual we mean essentially that

(a) $X_{d}\left[j_{i}^{n}, j_{i+1}^{n}\right)$ contains two deep traps, and so $\lambda_{3}<j_{i+1}^{n}$,

(b) $X_{d}$ exits $\mathbb{D}(n)$ before $j_{i+1}^{n}$, and so $\Lambda_{d}<j_{i+1}^{n}$,

(c) $X_{d}$ returns to the first deep trap after exiting a disk of area $2^{n} n^{-\kappa}$ around it, i.e. again $\lambda_{3}<j_{i+1}^{n}$,

(d) Disk $D_{y}\left(2^{n} n^{-\kappa}\right)$ intersects the complement of $D_{x_{i}^{n}}\left(2^{n} n^{\gamma}\right)$, i.e. $X_{d}$ hits a deep trap that is too close to the border of $D_{x_{i}^{n}}\left(2^{n} n^{\gamma}\right)$.

We will study the behaviour of the trajectory of the process

$$
\hat{Y}^{n}(t)=\frac{1}{2^{n / \alpha}} \sum_{i=0}^{\left\lfloor t n^{1-\alpha-\gamma}\right\rfloor} s_{i}^{n} .
$$

The value of this process becomes infinite if any of the possibilities from the previous paragraph happen. Therefore, we need to redefine $\hat{Y}^{n}$ slightly. Let $J_{1}(n)$ be the index of the first part of the trajectory where $s_{i}^{n}$ is infinite, $J_{1}(n)=\min \left\{i: s_{i}^{n}=\infty\right\}$. For technical reasons we introduce another three bad events. Let

$$
J_{2}(n)=\min \left\{i: x_{i+1}^{n} \notin \mathcal{E}(n)\right\},
$$

that means that the end of the $J_{2}$-th part of the trajectory is too close to some deep trap. The reason why we introduce this time is that when a part of the trajectory starts too close to some deep trap, the chance of hitting this trap is large, and thus the value of the score is strongly influenced by the mean waiting time of this trap.

For similar reasons we introduce

$$
J_{3}(n)=\min \left\{i: \operatorname{dist}\left(x_{i}^{n}, \mathbb{D}(n)^{c}\right) \leq \sqrt{\pi^{-1} 2^{n} n^{\gamma}}\right\},
$$

i.e. the $J_{3}$-th part is the first part that starts too close to the border of $\mathbb{D}(n)$ and $X$ can therefore exit from the large disk during it.

Further, let

$$
J_{4}(n)=\min \left\{i: X_{d}\left[0, j_{i}^{n}\right) \cap T_{\varepsilon}^{M} \cap X_{d}\left[j_{i}^{n}, j_{i+1}^{n}\right) \neq \emptyset\right\},
$$

which means that $X_{d}$ returns during part $J_{4}$ to some deep trap visited in previous parts of the trajectory. Let $J(n)=\min \left\{J_{1}(n), \ldots, J_{4}(n)\right\}$. The value of $J$ is the index of the first part of the trajectory where at least one of the following bad events happens 
(i) $X_{d}$ visits two different deep traps

(ii) $X_{d}$ can exit $\mathbb{D}(n)$

(iii) $X_{d}$ returns to some deep trap $y$ (possibly visited in previous parts) after exiting $D_{y}\left(2^{n} n^{-\kappa}\right)$

(iv) the end of this part of trajectory is too close (in the sense of (23)) to some deep trap.

(v) $X_{d}$ hits a deep trap that is too close to the border of $D_{x_{i}^{n}}\left(2^{n} n^{\gamma}\right)$.

Note that (iii) includes (c) from the previous list, (ii) contains (b), and (i), (v) is same as (a), (d).

Let now $\tilde{s}_{i}^{n}$ be a suitably chosen collection of i.i.d. random variables whose distribution will be defined later (see proof of Proposition 7.1). We set

$$
\bar{s}_{i}^{n}= \begin{cases}s_{i}^{n} & \text { if } i<J(n), \\ \tilde{s}_{i}^{n} & \text { otherwise. }\end{cases}
$$

Instead of the process $\hat{Y}^{n}$ we will use

$$
Y^{n}(t)=\frac{1}{2^{n / \alpha}} \sum_{i=0}^{\left\lfloor t n^{1-\alpha-\gamma}\right\rfloor} \bar{s}_{i}^{n} .
$$

We want to compare this process with the properly rescaled time change $S(n)$, namely with

$$
\bar{S}^{n}(t)=\frac{1}{2^{n / \alpha}} S\left(j_{\left\lfloor t n^{1-\alpha-\gamma}\right.}^{n}\right)
$$

To this end we should control several quantities. First, we should estimate the time spent in the shallow traps, that is in $T^{\varepsilon}$ (Section 3). Second, we need to control the probability that $X_{d}$ hits $T_{M}$ before $\Lambda_{d}$, because we did not include the very deep traps into the definition of the score (Section 4). Finally, we need to be sure that the value of $J$ is large enough, otherwise the process $Y^{n}$ has no relevance for our model (Section 5).

If all these conditions are satisfied, that means that $Y^{n}$ is a good approximation of $\bar{S}_{n}$ at least at the start of the trajectory, then we should study the behaviour of the sequence $Y^{n}$. We will show that it converges to a certain Lévy process (Section 6).

\section{The shallow traps}

As we already noted in the previous section, we want to show that the proportion of time that $X$ spends in the shallow traps is negligible. It will be shown later that the time that $X$ needs to leave disk $\mathbb{D}(n)$ is of order $2^{n / \alpha}$. We thus need to prove that the time spent in $T^{\varepsilon}$ can be made arbitrarily small with respect to $2^{n / \alpha}$. This is the result of the following lemma, whose proof occupies the rest of this section. 
Lemma 3.1. There exists $K_{1}=K_{1}(m)$ independent of $\varepsilon$ such that for $\mathbb{P}$-a.e. random environment $\boldsymbol{\tau}$ and for $n$ large enough

$$
\mathbb{E}\left[\sum_{i=0}^{\Lambda_{d}-1} e_{i} \tau_{X_{d}(i)} \chi\left\{X_{d}(i) \in T^{\varepsilon}\right\} \mid \tau\right] \leq K_{1} \varepsilon^{1-\alpha} 2^{n / \alpha} .
$$

Recall that $\Lambda_{d}$ is the first time that the discrete time process $X_{d}$ leaves the disk $\mathbb{D}(n)$. To prove this lemma we first describe the distribution of the shallow traps in the disk $\mathbb{D}(n)$. We divide the shallow traps into several groups. Let $i_{0}(n)$ be the integer satisfying $1 \leq \varepsilon 2^{-i_{0}(n)} \frac{2^{n / \alpha}}{n}<2$. For any $i \in\left\{1, \ldots, i_{0}(n)\right\}$, recall that

$$
T_{\varepsilon 2^{-i}}^{\varepsilon 2^{-i+1}}=\left\{x \in \mathbb{D}(n): \varepsilon 2^{-i} \frac{2^{n / \alpha}}{n} \leq \tau_{x}<\varepsilon 2^{-i+1} \frac{2^{n / \alpha}}{n}\right\} .
$$

Let $C$ be a large positive constant. We use $H_{1}=H_{1}(n, C, \varepsilon)$ to denote the event

$$
H_{1}(n, C, \varepsilon)=\left\{\left|T_{\varepsilon 2^{-i}}^{\varepsilon 2^{-i+1}}\right| \leq C n \varepsilon^{-\alpha} 2^{i \alpha}, \forall i \in\left\{1, \ldots, i_{0}(n)\right\}\right\} .
$$

We show that $H_{1}$ occurs with an overwhelming probability.

Lemma 3.2. There exists $K_{2}$ independent of $\varepsilon$ such that for $n$ large enough and for some positive constants $C$ and $c$.

$$
\mathbb{P}\left[H_{1}\left(n, K_{2}, \varepsilon\right)\right] \geq 1-C n \exp (-c n) .
$$

The proof is postponed.

Convention. At this place it is convenient to introduce one convention. Later in this paper we will need different properties of the environment that we will denote $H_{i}, i=1,2, \ldots$ For all these properties we will prove a result that allows an application of Borel-Cantelli lemma. When we prove such result we will suppose that these properties are verified. We thus may ignore a set of "unusual" environments whose probability is zero .

Proof of Lemma 3.1. The proof is divided into two parts. We first bound the time spent in "very" shallow traps: let $\xi$ be large enough such that

$$
(1-\xi)(1-\alpha)+1<0 .
$$

We define the set $\mathcal{S}$ of very shallow traps by

$$
\mathcal{S}=\left\{x \in \mathbb{D}(n): \tau_{x} \leq 2^{n / \alpha} n^{-\xi} \ll \varepsilon 2^{n / \alpha} / n\right\} .
$$

Let $G_{\mathbb{D}(n)}(\cdot, \cdot)$ denote the Green's function of the discrete time simple random walk in the disk $\mathbb{D}(n)$. Then we have

$$
\mathbb{E}\left[\sum_{j=0}^{\Lambda_{d}-1} e_{j} \tau_{X_{d}(j)} \chi\left\{X_{d}(j) \in \mathcal{S}\right\} \mid \tau\right]=\sum_{x \in \mathbb{D}(n)} G_{\mathbb{D}(n)}(0, x) \tau(x) \chi\{x \in \mathcal{S}\},
$$


Green's function can be bounded by (see (226) in Appendix A)

$$
G_{\mathbb{D}(n)}(0, x) \leq c n \quad \text { for all } x \in \mathbb{D}(n) .
$$

We thus have

$$
\mathbb{E}\left[\sum_{j=0}^{\Lambda_{d}-1} e_{j} \tau_{X_{d}(j)} \chi\left\{X_{d}(j) \in \mathcal{S}\right\} \mid \tau\right] \leq c n \sum_{x \in \mathbb{D}(n)} \tau(x) \chi\{x \in \mathcal{S}\} .
$$

Let $i_{1}(n)$ be the integer satisfying

$$
2^{-1+n / \alpha} n^{-\xi} \leq 2^{-i_{1}(n)} \varepsilon \frac{2^{n / \alpha}}{n} \leq 2^{n / \alpha} n^{-\xi},
$$

that is $i_{1}(n) \sim(\xi-1) \log _{2} n$. The expression (37) is bounded from above by

$$
c n \sum_{x \in \mathbb{D}(n)} \tau(x) \chi\{\tau(x) \leq 2\}+c n \sum_{i=i_{1}(n)}^{i_{0}(n)} \sum_{x \in \mathbb{D}(n)} \tau(x) \chi\left\{x \in T_{\varepsilon 2^{-i}}^{\varepsilon 2^{-i+1}}\right\} .
$$

By Lemma 3.2 and (33) this can be bounded by

$$
\begin{aligned}
& \leq 2 c n m 2^{n} n^{1-\alpha}+C n \sum_{i=i_{1}(n)}^{i_{0}(n)} \varepsilon 2^{-i+1} \frac{2^{n / \alpha}}{n} \cdot n \varepsilon^{-\alpha} 2^{i \alpha} \\
& \leq C n \varepsilon^{1-\alpha} 2^{n / \alpha} \sum_{i=i_{1}(n)}^{i_{0}(n)} 2^{i(\alpha-1)}+o\left(2^{n / \alpha}\right) \\
& \leq C \varepsilon^{1-\alpha} 2^{n / \alpha} n^{1+(1-\xi)(1-\alpha)}+o\left(2^{n / \alpha}\right)=o\left(2^{n / \alpha}\right) .
\end{aligned}
$$

This finishes the first part.

In the second part we bound the time spent in $T^{\varepsilon} \backslash \mathcal{S}$. We treat separately the time spent in $T_{\varepsilon 2^{-i}}^{\varepsilon 2^{-i+1}}$ for $i \in 1, \ldots, i_{1}(n)$, where $i_{1}(n)$ is defined as above. Let $K^{\prime}$ be a large positive constant and let $A(n, i)$ be the event

$$
A(n, i)=\left\{\sum_{x \in T_{\varepsilon 2^{-i}}^{\varepsilon 2^{-i+1}}} G_{\mathbb{D}(n)}(0, x) \tau(x) \geq K^{\prime} 2^{n / \alpha} \varepsilon^{1-\alpha} 2^{-i(1-\alpha)}\right\} .
$$

From the definition of $T_{\varepsilon 2^{-i}}^{\varepsilon 2^{-i+1}}$ we have

$$
\mathbb{P}[A(n, i)] \leq \mathbb{P}\left[2 \sum_{x \in T_{\varepsilon 2^{-i}}^{2^{-i+1}}} G_{\mathbb{D}(n)}(0, x) \geq K^{\prime} n \varepsilon^{-\alpha} 2^{\alpha i}\right]
$$

By Lemma 3.2, there are at most $K_{2} n \varepsilon^{-\alpha} 2^{i \alpha}$ sites in $T_{\varepsilon 2^{-i}}^{\varepsilon 2^{-i+1}} \mathbb{P}$-a.s. for large $n$. For $i=i_{1}(n)$ this number is of order $n^{1+\alpha(\xi-1)}$, for all others $i$ 's it is smaller.

Let $y_{i}, i=1, \ldots, R_{n}$, be a collection of uniformly, independently chosen points in $\mathbb{D}(n)$. By an easy combinatorial argument it is possible to prove that if 
$R_{n}$ is $o\left(2^{n / 2} n^{(1-\alpha) / 2}\right)$, then the probability that two of them are at the same place tends to zero. Since this is evidently satisfied for the number of sites in any of $T_{\varepsilon 2^{-i}}^{\varepsilon^{-i+1}}$, we can bound the sum in (42) by the sum over the random collection $y_{i}$, $i=1, \ldots, K_{2} n \varepsilon^{-\alpha} 2^{i \alpha}$. For any small, positive $c$ and for $n$ large enough we thus have

$$
\mathbb{P}[A(n, i)] \leq(1+c) \mathbb{P}\left[2 \sum_{i=1}^{K_{2} n \varepsilon^{-\alpha} 2^{i \alpha}} G_{\mathbb{D}(n)}\left(0, y_{i}\right) \geq K^{\prime} n \varepsilon^{-\alpha} 2^{\alpha i}\right]
$$

It is known that there exist constants $\lambda$ and $C$ not depending on $n$ such that (see Lemma A.2 for proof of this claim)

$$
\mathbb{E}\left[\exp \left(\lambda G_{\mathbb{D}(n)}\left(0, y_{1}\right)\right)\right] \leq C .
$$

By standard argument we can thus choose $K^{\prime}$ not depending on $i$ such that

$$
\mathbb{P}[A(n, i)] \leq c \exp \left(-c^{\prime} n \varepsilon^{-\alpha} 2^{i \alpha}\right) .
$$

Since $i_{1}(n) \ll n$, we get by summation

$$
\mathbb{P}\left[\bigcup_{i=1}^{i_{1}(n)} A(n, i)\right] \leq c n \exp \left(-c^{\prime} n \varepsilon^{-\alpha}\right),
$$

and thus for $n$ large enough none of $A(n, i)$ occurs $\mathbb{P}$-a.s. However, if it is the case, we have (using also the result of the first part of the proof)

$$
\begin{aligned}
\mathbb{E}\left[\sum_{j=0}^{\Lambda_{d}-1} e_{j} \tau_{X_{d}(j)} \chi\left(X_{d}(j) \in T^{\varepsilon}\right) \mid \boldsymbol{\tau}\right] \\
\leq \sum_{i=0}^{i_{1}(n)} K^{\prime} 2^{n / \alpha} \varepsilon^{1-\alpha} 2^{-i(1-\alpha)}+o\left(2^{n / \alpha}\right) \leq K_{1} 2^{n / \alpha} \varepsilon^{1-\alpha}
\end{aligned}
$$

This finishes the proof.

It remains to show Lemma 3.2.

Proof of Lemma 3.2. We first study the size of $T_{\varepsilon 2^{-i}}^{\varepsilon 2^{-i+1}}$ for some fixed index $i$. The probability $p_{n, i}$ that a site in $\mathbb{D}$ is in $T_{\varepsilon 2^{-i}}^{\varepsilon 2^{-i+1}}$ is

$$
p_{n, i}=\varepsilon^{-\alpha} \frac{n^{\alpha}}{2^{n}} 2^{i \alpha}\left[L\left(\varepsilon 2^{-i} \frac{2^{n / \alpha}}{n}\right)-\left(\frac{1}{2}\right)^{\alpha} L\left(\varepsilon 2^{-i+1} \frac{2^{n / \alpha}}{n}\right)\right] .
$$

Recall that $L$ defined in (16) is bounded, so the expression in the brackets can be bounded from above uniformly in $i$ by some constant depending only on the function $L$. Hence,

$$
p_{n, i} \leq c \varepsilon^{-\alpha} \frac{n^{\alpha}}{2^{n}} 2^{i \alpha}
$$


Applying exponential Markov bound we get for $\lambda>0$, using (49) and the fact that $(1+1 / n)^{n} \leq e$

$$
\begin{aligned}
\mathbb{P}\left[\left|T_{\varepsilon 2^{-i}}^{\varepsilon 2^{-i+1}}\right| \geq K_{2} n \varepsilon^{-\alpha} 2^{i \alpha}\right] & \leq \exp \left(-\lambda K_{2} n \varepsilon^{-\alpha} 2^{i \alpha}\right) \mathbb{E}\left[\exp \left(\lambda\left|T_{\varepsilon 2^{-i}}^{\varepsilon 2^{-i+1}}\right|\right)\right] \\
& =\exp \left(-\lambda K_{2} n \varepsilon^{-\alpha} 2^{i \alpha}\right)\left[\left(1-p_{n, i}\right)+p_{n, i} e^{\lambda}\right]^{m 2^{n} n^{1-\alpha}} \\
& \leq \exp \left[n \varepsilon^{-\alpha} 2^{i \alpha}\left(-K_{2} \lambda+m c e^{\lambda}\right)\right]
\end{aligned}
$$

If $K_{2}$ is chosen large enough, the expression in the parentheses is negative and thus the required probability decreases exponentially. The probability of $H_{1}^{c}$ satisfies

$$
\begin{aligned}
\mathbb{P}\left[H_{1}^{c}\right] & =\mathbb{P}\left[\bigcup_{i=1}^{i_{0}(n)}\left(\left|T_{\varepsilon 2^{-i}}^{\varepsilon 2^{-i+1}}\right| \geq K_{2} n \varepsilon^{-\alpha} 2^{i \alpha}\right)\right] \\
& \leq \sum_{i=1}^{i_{0}(n)} \exp \left\{n \varepsilon^{-\alpha} 2^{i \alpha}\left(-K_{2} \lambda+m c e^{\lambda}\right)\right\} \\
& \leq i_{0}(n) \exp \left\{n \varepsilon^{-\alpha}\left(-K_{2} \lambda+m c e^{\lambda}\right)\right\} .
\end{aligned}
$$

Since $i_{0}(n) \leq n / \alpha$, the proof is finished.

\section{Very deep traps}

In this section we estimate the probability of hitting a very deep trap. The aim is to show that these sites may be neglected from the analysis.

Lemma 4.1. For every $\delta>0$ and $m$ there exists $M$ such that for $n$ large enough and for $\mathbb{P}$-a.e. environment $\boldsymbol{\tau}$

$$
\mathbb{P}\left[X_{d}(t) \text { hits } T_{M}(n) \text { before } \Lambda_{d}(n) \mid \tau\right] \leq \delta .
$$

Proof. The standard large deviation argument gives

$$
\mathbb{P}\left[\left|T_{M}(n)\right|>C n m / M^{\alpha}\right] \leq C^{\prime} \exp \left(-c n m / M^{\alpha}\right)
$$

for some constants $C, C^{\prime}$ and $c$. We can thus take $\mathbb{P}$-a.s. $n$ large enough such that $\left|T_{M}(n)\right| \leq C n m / M^{\alpha}$. Let $A$ be an uniformly chosen random subset of $\mathbb{D}(n)$ with $\mathrm{Cnm} / \mathrm{M}^{\alpha}$ elements. Then

$$
\mathbb{P}\left[\mathbb{P}\left[X_{d} \text { hits } T_{M} \text { before } \Lambda_{d} \mid \tau\right]>\delta\right] \leq \mathbb{P}\left[\mathbb{P}\left[X_{d} \text { hits } A \text { before } \Lambda_{d} \mid A\right]>\delta\right] .
$$

Further, let $\left\{y_{i}\right\}, i=1, \ldots, C n m / M^{\alpha}$ be a collection of independently, uniformly chosen random points in $\mathbb{D}(n)$. As in the previous section we can replace $A$ by this collection. The expression (54) is then bounded by

$$
\leq(1+c) \mathbb{P}\left[\sum_{i=1}^{C n m / M^{\alpha}} \mathbb{P}\left[X_{d} \text { hits } y_{i} \text { before } \Lambda_{d} \mid y_{i}\right] \geq \delta\right]
$$


for some small positive $c$. Since the terms in the summation are independent, we can bound the last expression, using again the exponential Markov inequality, by

$$
\leq(1+c) \exp \left(-\delta \lambda_{n}\right) \mathbb{E}\left[\exp \left(\lambda_{n} \mathbb{P}\left[X_{d} \text { hits } y_{i} \text { before } \Lambda_{d} \mid y_{i}\right]\right)\right]^{C n m / M^{\alpha}} .
$$

The inequality (228) from Appendix A applied on the disk $\mathbb{D}(n)$ gives

$$
\mathbb{E}\left[\exp \left(-(n \log 2 / 2+o(n)) \mathbb{P}\left[X_{d} \text { hits } y_{1} \text { before } \Lambda_{d}\right]\right)\right] \leq C .
$$

Therefore, taking $\lambda_{n}=b \log \sqrt{\pi^{-1} m 2^{n} n^{1-\alpha}}, b<1$

$$
\mathbb{P}\left[\mathbb{P}\left[X_{d} \text { hits } T_{M} \text { before } \Lambda_{d} \mid \tau\right]>\delta\right] \leq \exp \left\{-\delta c n+c^{\prime} m n / M^{\alpha}+o(n)\right\} .
$$

The lemma then follows by taking $M$ large enough and applying Borel-Cantelli argument.

\section{5. $J$ is large enough}

To justify the approximation of $\bar{S}^{n}$ by $Y^{n}$ we should now prove that the index of the first bad part, $J$, is large enough. More precisely, we should show that one can choose $\kappa$ and $m$ such that, with large probability, the index $J$ of the first bad part of the trajectory of $X$ is sufficiently large for our purposes.

Lemma 5.1. For any $\delta, k$, and $\mathbb{P}$-a.e. $\tau$ there exist $m$ and $\kappa$ not depending on $\varepsilon$ and $M$ such that for $n$ large enough

$$
\mathbb{P}\left[J(n) n^{\alpha+\gamma-1} \geq k \mid \tau\right] \geq 1-\delta .
$$

To prove this lemma we should verify that all events described in Section 2 happen with low probability. This is the goal of all following technical lemmas. The proof of Lemma 5.1 can be found at the end of this section.

Event (i). The most complicated part of the proof is to show that $X$ does not hit two deep traps during one part of the trajectory. The following lemma is a little bit more precise than is needed to bound $J$, however, we will need this more precise result later. We use $p_{\varepsilon}^{M}$ to denote the factor $\varepsilon^{-\alpha}-M^{-\alpha}$.

Lemma 5.2. Let

$$
V_{x_{0}}(n)=\sum_{y \in T_{\varepsilon}^{M}} \mathbb{P}_{x_{0}}\left[X_{d} \text { hits y before exiting } D_{x_{0}}\left(2^{n} n^{\gamma}\right) \mid \boldsymbol{\tau}\right],
$$

where $\mathbb{P}_{x_{0}}$ denotes the law of the simple random walk $X_{d}$ started at $x_{0}$. Then for any $\delta$ and $\mathbb{P}$-a.e. $\tau$ there exists $n_{0}$ such that for all $n>n_{0}$ and $x_{0} \in \mathcal{E}(n)$ (see (15) for definition of $\mathcal{E}(n)$ ),

$$
\frac{\mathcal{K}(1-\delta) p_{\varepsilon}^{M}}{n^{1-\alpha-\gamma}} \leq V_{x_{0}}(n) \leq \frac{\mathcal{K}(1+\delta) p_{\varepsilon}^{M}}{n^{1-\alpha-\gamma}}
$$

with $\mathcal{K}=(\log 2)^{-1}$. 
To prove this lemma we should describe the distribution of the deep traps inside $\mathbb{D}(n)$. This description is contained in Lemmas 5.3 and 5.4.

First, we will show that the deep traps are distributed almost homogeneously around the disk. Let $v<\gamma<1-\alpha$ and let $H_{2}=H_{2}(n, \delta, v, \varepsilon, M)$ be the set of configurations of the environment satisfying the "homogeneity" condition:

$$
\begin{aligned}
H_{2}=\{\mid & T_{\varepsilon}^{M} \cap B_{x}\left(2^{n} n^{\nu}\right) \mid \in\left[(1-\delta) p_{\varepsilon}^{M} n^{\nu+\alpha},(1+\delta) p_{\varepsilon}^{M} n^{\nu+\alpha}\right] \\
& \left.\quad \text { for all } x \text { such that } B_{x}\left(2^{n} n^{\nu}\right) \subset \mathbb{D}(n) .\right\}
\end{aligned}
$$

Lemma 5.3. For any $\varepsilon, M$, and $\delta$ there exist positive constants $C$ and $c$ such that for $n$ large enough

$$
\mathbb{P}\left[H_{2}\right] \geq 1-C n^{1-\alpha-v} \delta^{-2} \exp \left(-c n^{\nu+\alpha}\right) .
$$

Proof. We divide the complement of $\mathrm{H}_{2}$ into two parts. First, we treat the case when there is a region in $\mathbb{D}$ where there are not enough deep traps. Let $A$ be the event that there is a square of area $2^{n} n^{\nu}$ in $\mathbb{D}(n)$ where there are less than $(1-\delta) p_{\varepsilon}^{M} n^{\nu+\alpha}$ sites from $T_{\varepsilon}^{M}(n)$,

$$
A=\left\{\exists x \in \mathbb{D}:\left|T_{\varepsilon}^{M} \cap B_{x}\left(2^{n} n^{\nu}\right)\right|<(1-\delta) p_{\varepsilon}^{M} n^{\nu+\alpha}, D_{x}\left(2^{n} n^{\nu}\right) \subset \mathbb{D}\right\} .
$$

We use $G$ to denote the grid $\left\lfloor 2^{n / 2} n^{\nu / 2} \delta / 5\right\rfloor \mathbb{Z}^{2}$. Every square of area $2^{n} n^{v}$ contains at least one square of area $2^{n} n^{v}(1-\delta / 2)$ with the centre in $G$ for $n$ sufficiently large. Hence, if $A$ is true, then there is a square of area $2^{n} n^{v}(1-\delta / 2)$ which has centre $x \in G$, and which contains less than $(1-\delta) p_{\varepsilon}^{M} n^{\nu+\alpha}$ sites. We use $A_{x}$ to denote the last event. We have

$$
\mathbb{P}[A] \leq \sum_{x} \mathbb{P}\left[A_{x}\right]=C^{\prime} \delta^{-2} n^{1-\alpha-v} \mathbb{P}\left[A_{x}\right],
$$

where the sum runs over all $x \in G$ such that $B_{x}\left((1-\delta / 2) 2^{n} n^{v}\right) \subset \mathbb{D}$. We used the obvious fact that $\mathbb{P}\left[A_{x}\right]$ does not depend on $x$. The probability of $A_{x}$ can be bounded using standard methods. Take $\eta>0$. For $n$ large enough, the probability $p$ that a site is in $T_{\varepsilon}^{M}(n)$ is larger than $(1-\eta) p_{\varepsilon}^{M} 2^{-n} n^{\alpha}$. For $\lambda>0$ we have

$$
\begin{aligned}
& \mathbb{P}\left[A_{x}\right] \leq \exp \left(\lambda(1-\delta) n^{\nu+\alpha} p_{\varepsilon}^{M}\right)\left[(1-p)+e^{-\lambda} p\right]^{2^{n} n^{\nu}(1-\delta / 2)} \\
& \quad \leq \exp \left(\lambda(1-\delta) n^{\nu+\alpha} p_{\varepsilon}^{M}\right)\left[1+\left(e^{-\lambda}-1\right) \frac{(1-\eta) n^{\alpha} p_{\varepsilon}^{M}}{2^{n}}\right]^{2^{n} n^{\nu}\left(1-\frac{\delta}{2}\right)} .
\end{aligned}
$$

If $n$ is large enough, the last expression is bounded by

$$
\mathbb{P}\left[A_{x}\right] \leq \exp \left[n^{\nu+\alpha} p_{\varepsilon}^{M}\left(\lambda(1-\delta)+\left(e^{-\lambda}-1\right)(1-\eta)^{2}(1-\delta / 2)\right)\right] .
$$

It is not difficult to show that for any $\delta$ there exist $\eta$ and $\lambda$ such that the exponent is negative. Hence, we have

$$
\mathbb{P}[A] \leq C^{\prime} n^{1-\alpha-v} \delta^{-2} \exp \left(-c^{\prime} n^{\nu+\alpha}\right) .
$$

In the second part of the proof we exclude the possibility that there are places in $\mathbb{D}$ where the deep traps are too dense. Let $B$ be the event that there is a square of 
area $2^{n} n^{\nu}$ intersecting $\mathbb{D}(n)$ where is more than $(1+\delta) \varepsilon^{-\alpha} n^{\nu+\alpha}$ sites from $T_{\varepsilon}^{M}(n)$. The probability of $B$ can be bounded exactly in the same way as the probability of $A$, one should only consider the squares with area $2^{n} n^{v}(1+\delta / 2)$ and centres in $G$. We thus have

$$
\mathbb{P}\left[H_{2}(n)^{c}\right] \leq \mathbb{P}[A \cup B] \leq C n^{1-\alpha-v} \delta^{-2} \exp \left(-c n^{\nu+\alpha}\right) .
$$

This finishes the proof.

The lemma we have just proved is not precise enough to bound the probability of hitting traps that are closer than $\sqrt{2^{n} n^{v}}$ to the starting point. The following lemma will serve us for that bound. Again, it describes some sort of homogeneity of the environment

We consider the events $H_{3}(i)=H_{3}(i, n, \kappa, \varepsilon, M)$,

$$
H_{3}(i)=\left\{\exists x \in \mathbb{D}(n):\left|B_{x}\left(2^{n+i} n^{-\kappa}\right) \cap T_{\varepsilon}^{M}\right| \leq 4 \log ^{2} n\left(1 \vee 2^{i} n^{\alpha-\kappa} \varepsilon^{-\alpha}\right)\right\},
$$

where $a \vee b$ denotes the maximum of $a, b$. We define $H_{3}$ by

$$
H_{3}=\bigcap_{i=-1}^{\infty} H_{3}(i) \text {. }
$$

Observe that $2^{n+i} n^{-\kappa} \ll 2^{n} n^{v}$ for fixed $i$ and $n$ large enough. So, we study here much smaller squares than in the previous lemma. Hence, the description of the homogeneity is more precise in this direction. On the other hand, we prove only the upper bound on the number of the deep traps in these squares and this bound is also "weaker" than the previous bound.

Lemma 5.4. There exists a constant $C$ such that

$$
\mathbb{P}\left[\mathrm{H}_{3}\right] \geq 1-\mathrm{Cn}^{-3} \text {. }
$$

Proof. Fix some $i$ and consider the lattice $G_{i}=\mathbb{Z}^{2} \sqrt{2^{n+i} n^{-\kappa}}$. If there is $x$ such that $\left|B_{x}\left(2^{n+i} n^{-\kappa}\right) \cap T_{\varepsilon}^{M}\right| \geq 4 \log ^{2} n\left(1 \vee 2^{i} n^{\alpha-\kappa} \varepsilon^{-\alpha}\right)$, then there is a point $y \in G_{i}$ such that $B_{y}\left(4 \cdot 2^{n+i} n^{-\kappa}\right)$ contains more than $4 \log ^{2} n\left(1 \vee 2^{i} n^{\alpha-\kappa} \varepsilon^{-\alpha}\right)$ sites from $T_{\varepsilon}^{M}$. The number of squares with area $4 \cdot 2^{n+i} n^{-\kappa}$ and centres in $G_{i}$ that intersect $\mathbb{D}(n)$ is bounded by $C n^{1-\alpha+\kappa} 2^{-i}$.

Consider now one such square. The probability that it contains too many sites from $T_{\varepsilon}^{M}$ can be bounded by standard arguments

$$
\begin{aligned}
& \mathbb{P}\left[\left|B\left(4 \cdot 2^{n+i} n^{-\kappa}\right) \cap T_{\varepsilon}^{M}\right| \geq 4 \log ^{2} n\left(1 \vee 2^{i} n^{\alpha-\kappa} \varepsilon^{-\alpha}\right)\right] \\
& \quad \leq c \exp \left(-\lambda 4 \log ^{2} n\left(1 \vee 2^{i} n^{\alpha-\kappa} \varepsilon^{-\alpha}\right)+4 p_{\varepsilon}^{M}\left(e^{\lambda}-1\right) 2^{i} n^{\alpha-\kappa} \varepsilon^{-\alpha}\right) .
\end{aligned}
$$

Since $\alpha-\kappa<0$, we can choose $\lambda$ such that for $n$ large enough the last expression is bounded by $(1 / 2)^{\log ^{2} n}$. Summation over $i$ and over all squares that intersect $\mathbb{D}(n)$ gives us

$$
\mathbb{P}\left[H_{6}^{c}\right] \leq \sum_{i=-1}^{\infty} C 2^{-i} n^{1-\alpha+\kappa}(1 / 2)^{\log ^{2} n} \leq C n^{-3}
$$


We now have all ingredients to prove Lemma 5.2.

Proof of Lemma 5.2. We can suppose that $x_{0}$ is the origin. We use $\xi$ to denote the exit time from $D\left(2^{n} n^{\gamma}\right)$. Let $\gamma^{\prime}$ be a constant satisfying $v<\gamma^{\prime}<\gamma$. We divide the sum $V_{0}(n)$ into two parts. First, we sum over all deep traps that are far enough from the origin. Precisely, we consider the deep traps that are in $D\left(2^{n} n^{\gamma}\right) \backslash D\left(2^{n} n^{\gamma^{\prime}}\right)$. Let $I_{1}$ denotes the sum over such traps. We use $I_{2}$ to denote the sum over the remaining deep traps.

To show the upper bound on $I_{1}$, we cover the set $D\left(2^{n} n^{\gamma}\right) \backslash D\left(2^{n} n^{\gamma^{\prime}}\right)$ by squares of area $2^{n} n^{v}$ and centres in $\sqrt{2^{n} n^{v}} \mathbb{Z}^{2}$. Let $x_{1}, \ldots, x_{R}$ denote the set of centres of such squares that intersect $D\left(2^{n} n^{\gamma}\right) \backslash D\left(2^{n} n^{\gamma^{\prime}}\right)$. Since $v<\gamma^{\prime}$, the size of each such square is negligible with respect to its distance to the origin. All deep traps in such squares have thus almost the same chance to be hit. We use expression (225) from Appendix A to estimate probability that $X$ hits some point before exiting from $D\left(2^{n} n^{\gamma}\right)$. Let $r_{n}$ be the radius of this disk, $r_{n}=\sqrt{\pi^{-1} 2^{n} n^{\gamma}}$.

$$
\begin{aligned}
I_{1} & \leq \sum_{i=1}^{R} \sum_{\substack{y_{j} \in B_{x_{i}}\left(2^{n_{n}} n^{v}\right) \\
y_{j} \in T_{\varepsilon}^{M}}}\left(1-\frac{\log \left|y_{j}\right|}{\log r_{n}}+O\left(n^{-2}\right)\right) \\
& =\sum_{i=1}^{R}\left|B_{x_{i}}\left(2^{n^{v}} n^{v}\right) \cap T_{\varepsilon}^{M}\right|\left(1-\frac{\log \left|x_{i}\right|}{\log r_{n}}+O\left(n^{-1+\left(v-\gamma^{\prime}\right) / 2}\right)\right)
\end{aligned}
$$

where we use the estimate

$$
\frac{\log \left|y_{j}\right|}{\log r_{n}}-\frac{\log \left|x_{i}\right|}{\log r_{n}}=O\left(n^{-1+\left(v-\gamma^{\prime}\right) / 2}\right)
$$

that is valid for any $y_{j} \in B_{x_{i}}\left(2^{n} n^{v}\right)$.

From Lemma 5.3 we know that for $n$ large enough $\left|B_{x_{i}}\left(2^{n} n^{\nu}\right) \cap T_{\varepsilon}^{M}\right| \leq$ $n^{\nu+\alpha} p_{\varepsilon}^{M}(1+\delta / 2)$ and thus

$$
I_{1} \leq \sum_{i=1}^{R} n^{\nu+\alpha} p_{\varepsilon}^{M}(1+\delta / 2)\left(1-\frac{\log \left|x_{i}\right|}{\log r_{n}}+O\left(n^{-1+\left(\nu-\gamma^{\prime}\right) / 2}\right)\right) .
$$

We now replace the summation by integration making again an error of order $O\left(n^{-1+\left(\nu-\gamma^{\prime}\right) / 2}\right) . I_{1}$ is thus bounded from above by

$$
\int_{D\left(2^{n} n \gamma\right) \backslash D\left(2^{n} n \gamma^{\prime}\right)} \frac{n^{\nu+\alpha} p_{\varepsilon}^{M}}{2^{n} n^{\nu}}\left(1+\frac{\delta}{2}\right)\left(1-\frac{\log |x|}{\log r_{n}}+O\left(n^{-1+\left(\nu-\gamma^{\prime}\right) / 2}\right)\right) d x .
$$

The integration gives

$$
I_{1} \leq \frac{n^{\alpha+\gamma-1} p_{\varepsilon}^{M}}{\log 2}\left(1+\frac{\delta}{2}\right)(1+o(1)) \leq \frac{n^{\alpha+\gamma-1} p_{\varepsilon}^{M}}{\log 2}\left(1+\frac{3 \delta}{4}\right)
$$


for $n$ large enough. This finishes the proof of the upper bound for $I_{1}$. The proof of the lower bound is analogous. After a very similar calculation we get

$$
I_{1} \geq \frac{n^{\alpha+\gamma-1} p_{\varepsilon}^{M}}{\log 2}\left(1-\frac{3 \delta}{4}\right) .
$$

We should now estimate the sum $I_{2}$ over all sites $x \in T_{\varepsilon}^{M} \cap\left(D\left(2^{n} n^{\gamma^{\prime}}\right) \backslash\right.$ $\left.D\left(2^{n} n^{-\kappa}\right)\right)$. The disk $D\left(2^{n} n^{-\kappa}\right)$ can be excluded since by the assumptions of the lemma $x \in \mathcal{E}(n)$ and so there are no deep traps in this disk. We cover the domain by objects comprising eight squares of area $2^{n+i} n^{-\kappa}$ whose union is the square, centred at the origin, of nine times larger area with the middle square cut off. The parameter $i$ takes values in the set $\left\{-1,0,1, \ldots,\left(\gamma^{\prime}+\kappa\right) \log _{2} n\right\}$. We use this covering because if the trap is too close to the origin, we should know more precisely its position to estimate its hitting probability. Our covering becomes clearly finer when the origin is approached.

Any point inside the $i$-th object from the previous paragraph has distance from the origin at least $\sqrt{2^{n+i} n^{-\kappa}} / 2$. In each of the eight squares there is, by Lemma 5.4, at most $4 \log ^{2} n\left(1 \vee 2^{i} n^{\alpha-\kappa} \varepsilon^{-\alpha}\right)$ sites from $T_{\varepsilon}^{M}$. By formula (225) for the hitting probability of a point in $\mathbb{D}\left(2^{n} n^{\gamma}\right)$ we have

$$
\begin{aligned}
I_{2} \leq & 8 \sum_{i=-1}^{\left(\gamma^{\prime}+\kappa\right) \log _{2} n}\left[1-\frac{\log \left(\sqrt{2^{n+i} n^{-\kappa}} / 2\right)}{\log r_{n}}+O\left(\frac{2^{-n-i} n^{\kappa}}{\log r_{n}}\right)+O\left(\log ^{-2} r_{n}\right)\right] \\
& \times 4 \log ^{2} n\left(1 \vee 2^{i} n^{\alpha-\kappa} \varepsilon^{-\alpha}\right) .
\end{aligned}
$$

The expression in the brackets can be easily bounded by $C n^{-1} \log n$ with some large constant $C$. Hence,

$$
I_{2} \leq C \sum_{i=-1}^{\left(\gamma^{\prime}+\kappa\right) \log _{2} n} \frac{\log n}{n} \log ^{2} n\left(1 \vee 2^{i} n^{\alpha-\kappa} \varepsilon^{-\alpha}\right)
$$

Since the expression inside of the summation is increasing in $i$, the last display can be trivially estimated by $\left(\gamma^{\prime}+\kappa\right) \log _{2} n$ times the last term. This gives

$$
I_{2} \leq C n^{\alpha+\gamma^{\prime}-1} \log ^{4} n \ll \frac{n^{\alpha+\gamma-1} p_{\varepsilon}^{M}}{\log 2}\left(1+\frac{\delta}{4}\right) .
$$

Putting together (79), (80), and (83) we get

$$
\frac{n^{\alpha+\gamma-1} p_{\varepsilon}^{M}}{\log 2}(1-\delta) \leq I_{1} \leq V_{0}(n)=I_{1}+I_{2} \leq \frac{n^{\alpha+\gamma-1} p_{\varepsilon}^{M}}{\log 2}(1+\delta) .
$$

This finishes the proof of Lemma 5.2.

Using exactly the same approach as above and Lemma 5.6 below we show 
Lemma 5.5. For $x \in T_{\varepsilon}^{M}$, let us redefine

$$
V_{x}(n)=\sum_{y \in T_{\varepsilon}^{M} \backslash\{x\}} \mathbb{P}_{x}\left[X_{d} \text { hits y before exiting } D_{x}\left(2^{n+1} n^{\gamma}\right) \mid \boldsymbol{\tau}\right],
$$

where $\mathbb{P}_{x}$ denotes the law of the simple random walk $X_{d}$ started at $x$. Then for any $\delta$ and $\mathbb{P}$-a.e. $\boldsymbol{\tau}$ there exists $n_{0}$ such that for all $n>n_{0}$ and all $x \in T_{\varepsilon}^{M}$,

$$
V_{x}(n) \leq \frac{C p_{\varepsilon}^{M}}{n^{1-\alpha-\gamma}} .
$$

Let $H_{4}=H_{4}(n, \kappa, \varepsilon)$ be the event

$$
H_{4}=\left\{\min \left\{|x-y|: x, y \in T_{\varepsilon}(n)\right\} \geq 2 \sqrt{\pi^{-1} 2^{n} n^{-\kappa}}\right\} .
$$

The constant 2 before the square root is not necessary for the current application, but it will be used later.

Lemma 5.6. There exists constant $C=C(\varepsilon, m)$ such that

$$
\mathbb{P}\left[H_{4}\right] \geq 1-C n^{1+\alpha-\kappa} .
$$

Proof. Let $B(x)$ be the event

$$
B(x)=\left\{x \in T_{\varepsilon}(n)\right\} \cap\left\{\exists y \in T_{\varepsilon}(n),|y-x| \leq 2 \sqrt{\pi^{-1} 2^{n} n^{-\kappa}}\right\} .
$$

Then

$$
\mathbb{P}[B(x)] \leq C \frac{n^{2 \alpha-\kappa}}{2^{n}} \varepsilon^{-2 \alpha} .
$$

and the result follows by summation over all $x \in \mathbb{D}(n)$.

The following lemma is an easy consequence of Lemma 5.2. It is the actual estimate of the probability of hitting a deep trap.

Lemma 5.7. For any $\delta>0$ and $\mathbb{P}$-a.e. $\boldsymbol{\tau}$, there exists $n_{0}$ such that for $n>n_{0}$ and for all $x \in \mathcal{E}(n)$, the probability that the simple random walk started at $x$ hits exactly one site from $T_{\varepsilon}^{M}(n)$ before exiting $D_{x}\left(2^{n} n^{\gamma}\right)$ is in interval

$$
\left(\mathcal{K}(1-\delta) p_{\varepsilon}^{M} n^{\alpha+\gamma-1}, \mathcal{K}(1+\delta) p_{\varepsilon}^{M} n^{\alpha+\gamma-1}\right) .
$$

The probability that it hits more than one deep trap is bounded by

$$
\mathbb{P}\left[X_{d} \text { hits at least two sites from } T_{\varepsilon}^{M}\right] \leq C n^{2(\alpha+\gamma-1)}\left(p_{\varepsilon}^{M}\right)^{2}
$$
for some positive constant $C$. 
Proof. Let $T_{\varepsilon}^{M} \cap D_{x}\left(2^{n} n^{\gamma}\right)=\left\{x_{1}, \ldots, x_{L}\right\}$. Assume that some point $x_{i}$ was hit by $X_{d}$ before the exit from $D\left(2^{n} n^{\gamma}\right)$.

We apply now Lemma 5.5 and the strong Markov property. We thus have

$$
\sum_{j \neq i} \mathbb{P}\left[X_{d} \text { hits } x_{j} \mid X_{d} \text { hit } x_{i}\right] \leq C n^{\alpha+\gamma-1} p_{\varepsilon}^{M} .
$$

The Bonferroni inequalities give

$$
\begin{aligned}
\mathbb{P}\left[X_{d} \text { hits } T_{\varepsilon}^{M}\right] & \leq \sum_{i} \mathbb{P}\left[X_{d} \text { hits } x_{i}\right] \leq \mathcal{K}(1+\delta) p_{\varepsilon}^{M} n^{\alpha+\gamma-1} \\
\mathbb{P}\left[X_{d} \text { hits } T_{\varepsilon}^{M}\right] & \geq \sum_{i} \mathbb{P}\left[X_{d} \text { hits } x_{i}\right]-\frac{1}{2} \sum_{i} \sum_{j \neq i} \mathbb{P}\left[X_{d} \text { hits } x_{i} \text { and } x_{j}\right] \\
& \geq \mathcal{K}(1-\delta) p_{\varepsilon}^{M} n^{\alpha+\gamma-1}-C\left(p_{\varepsilon}^{M}\right)^{2} n^{2(\alpha+\gamma-1)} \\
& \geq \mathcal{K}(1-2 \delta) p_{\varepsilon}^{M} n^{\alpha+\gamma-1}
\end{aligned}
$$

for $n$ large enough. Similarly we get from the strong Markov property and Lemma 5.5

$$
\mathbb{P}\left[X_{d} \text { hits at least two points from } T_{\varepsilon}^{M}\right] \leq C\left(p_{\varepsilon}^{M}\right)^{2} n^{2(\alpha+\gamma-1)} .
$$

This finishes the proof of Lemma 5.7.

Event (iv). To find a lower bound for $J$, we should further verify that the probability that a part of the trajectory ends too close to some deep trap is small.

Lemma 5.8. For $\mathbb{P}$-a.e. environment $\boldsymbol{\tau}$, the probability that the simple random walk started at arbitrary $x \in \mathbb{D}(n)$ exits $D_{x}\left(2^{n} n^{\gamma}\right)$ at some point that is in $\mathbb{D}(n) \backslash$ $\mathcal{E}(n)$ is smaller than $\mathrm{Cn}^{2-\kappa / 2-\gamma / 2}$.

Proof. We start again with the description of the properties of the environment. Let $r_{n}$ be the radius of the disk $D\left(2^{n} n^{\gamma}\right)$. We use $A_{x}\left(2^{n} n^{\gamma}\right)$ to denote the annular ring with the centre $x$, the inner radius $r_{n}-\sqrt{\pi^{-1} 2^{n} n^{-\kappa}}$, and the outer radius $r_{n}+\sqrt{\pi^{-1} 2^{n} n^{-\kappa}}$. Let $H_{5}=H_{5}(n, \varepsilon, M)$ be the event

$$
H_{5}=\left\{\left|T_{\varepsilon}^{M}(n) \cap A_{x}\left(2^{n} n^{\gamma}\right)\right| \leq n^{2} \text { for all } x \in \mathbb{D}(n)\right\} .
$$

Lemma 5.9. For $n$ large there exist constants $C$ and $c$ such that

$$
\mathbb{P}\left[H_{5}\right] \geq 1-C 2^{n} n^{1-\alpha} \exp \left(-c n^{2}\right) .
$$

Proof. There are less than $C 2^{n} n^{\gamma / 2-\kappa / 2}$ points in the annulus $A_{x}\left(2^{n} n^{\gamma}\right)$. The probability that a trap is in $T_{\varepsilon}^{M}(n)$ is of order $p_{\varepsilon}^{M} 2^{n} n^{-\alpha}$. The standard application of Markov inequality gives

$$
\mathbb{P}\left[\left|A_{x}\left(2^{n} n^{\nu}\right) \cap T_{\varepsilon}^{M}(n)\right|>n^{2}\right] \leq \exp \left(-c(\varepsilon, M) n^{2}\right) .
$$

The result follows by summation over all $x \in \mathbb{D}(n)$. 
We can now finish the proof of Lemma 5.8. We use the fact that probability of exiting the disk of radius $R$ in a particular point at its border is $O(1 / R)$ (see [Law91] Lemma 1.7.4). From Lemma 5.9 we know that there are less than $n^{2}$ deep traps in annulus $A_{x}\left(2^{n} n^{\gamma}\right)$. This implies that there are at most $c n^{2} \sqrt{2^{n} n^{-\kappa}}$ points on the border of $D_{x}\left(2^{n} n^{\gamma}\right)$ that are close to some deep trap. The required probability is thus bounded from above by

$$
C \sqrt{2^{-n} n^{-\gamma}} n^{2} \sqrt{2^{n} n^{-\kappa}}=C n^{2-\kappa / 2-\gamma / 2}
$$

This finishes the proof of Lemma 5.8.

Event (v). The next lemma excludes the possibility of hitting a deep trap that is too close to the border of the disk with area $2^{n} n^{\gamma}$ around the starting point.

Lemma 5.10. For any $x \in \mathbb{D}$, the probability that the random walk started at $x$ hits a deep trap in $A_{x}\left(2^{n} n^{\gamma}\right)$ before the exit from $D_{x}\left(2^{n} n^{\gamma}\right)$ is smaller than $C n^{2-\gamma / 2-\kappa / 2}$.

Proof. We need to estimate the probability that we hit some point $y$ that is in the distance smaller than $\sqrt{\pi^{-1} 2^{n} n^{-\kappa}}$ from the border of $D_{x}\left(2^{n} n^{\gamma}\right)$. We use (226) to estimate this probability. The advantage of (226) against (225) is that the error terms are much smaller. Since for any disk $D$ centred at $x$

$$
G_{D}(x, y)=\mathbb{P}_{x}\left[X_{d} \text { hits } y \text { before exit from } D\right] G_{D}(y, y)
$$

and $G_{D}(y, y) \geq 1$, we know that $\mathbb{P}_{x}\left(X_{d}\right.$ hits $\left.y\right) \leq G_{D}(x, y)$. According to Lemma 5.9 there are at most $n^{2}$ deep traps in $A_{x}\left(2^{n} n^{\gamma}\right)$. We thus have

$$
\begin{aligned}
& \mathbb{P}_{x}\left[X_{d} \text { hits } T_{\varepsilon}^{M} \cap A_{x}\left(2^{n} n^{\gamma}\right) \text { before exiting } D_{x}\left(2^{n} n^{\gamma}\right)\right] \\
& \leq \frac{2 n^{2}}{\pi}\left[\log \sqrt{\pi^{-1} 2^{n} n^{\gamma}}-\log \left(\sqrt{\pi^{-1} 2^{n} n^{\gamma}}\left(1-n^{-\gamma / 2-\kappa / 2}\right)\right)+O\left(2^{-n / 2}\right)\right] \\
& \leq-c n^{2} \log \left(1-n^{-\gamma / 2-\kappa / 2}\right) \leq C n^{2-\gamma / 2-\kappa / 2} .
\end{aligned}
$$

This finishes the proof.

Event (iii). Finally, we need to show that the process $X$ almost never returns to a deep trap after exiting a disk of area $2^{n} n^{-\kappa}$ around it. We do not need to consider the traps that are closer than $\sqrt{\pi^{-1} 2^{n} n^{-\kappa}}$ to the border of $\mathbb{D}$ because hitting such traps has already been dealt with when considering (ii) and (v) defining the "bad" event.

Lemma 5.11. There exists a constant $C$ such that for any site $x$ satisfying $D_{x}\left(2^{n} n^{-\kappa}\right)$ $\cap \mathbb{D}(n)^{c}=\emptyset$, the probability that the simple random walk returns to $x$ before $\Lambda_{d}$ after exiting disk $D_{x}\left(2^{n} n^{-\kappa}\right)$ is smaller than $C n^{-1} \log n$. 
Proof. Let $p_{\text {ret }}$ denote the required probability and let $\xi$ be the first time when $X_{d}$ exits $D_{x}\left(2^{n} n^{-\kappa}\right)$. Obviously, $\xi<\Lambda_{d}$. By the Markov property

$$
\begin{aligned}
G_{\mathbb{D}}(x, x) & =\sum_{i=0}^{\Lambda_{d}} \mathbb{P}_{x}\left[X_{d}(i)=x\right]=\sum_{i=0}^{\xi} \mathbb{P}_{x}\left[X_{d}(i)=x\right]+\sum_{i=\xi+1}^{\Lambda_{d}} \mathbb{P}_{x}\left[X_{d}(i)=x\right] \\
& =G_{D\left(2^{n} n^{-\kappa}\right)}(0,0)+p_{\text {ret }} G_{\mathbb{D}}(x, x) .
\end{aligned}
$$

Hence,

$$
p_{\text {ret }}=1-\frac{G_{D\left(2^{n} n^{-\kappa}\right)}(0,0)}{G_{\mathbb{D}}(x, x)} \leq 1-\frac{G_{D\left(2^{n} n^{-\kappa}\right)}(0,0)}{G_{2 \mathbb{D}}(0,0)},
$$

where $2 \mathbb{D}$ denotes the disk with centre the origin and twice the radius of $\mathbb{D}$. Using the expression (227) we get

$$
p_{\text {ret }} \leq 1-\frac{\log \left(2^{n} n^{-\kappa}\right)+O(1)}{\log \left(2 \cdot 2^{n} n^{1-\alpha}\right)+O(1)} \leq C n^{-1} \log n .
$$

This finishes the proof.

Proof of Lemma 5.1. We have now all ingredients to prove Lemma 5.1. We should prove that the probability that some of the events (i)-(v) from Section 2 happen during first $C n^{1-\alpha-\gamma}$ parts can be made very small. We will use $J_{(i)}, \ldots, J_{(v)}$ to denote the first part where (i), ..., resp. (v) occurs.

The simplest condition is (ii). This condition requires that $X$ cannot exit $\mathbb{D}$ during the good part of the trajectory. That means that starting point of a part of the trajectory satisfying (ii) should be in the annular ring with the outer radius $\sqrt{\pi^{-1} m 2^{n} n^{1-\alpha}}$ (which is the radius of $\mathbb{D}$ ) and the inner radius $\sqrt{\pi^{-1} m 2^{n} n^{1-\alpha}}-\sqrt{\pi^{-1} 2^{n} n^{\gamma}}$. The sequence of starting points $x_{i}^{n}$ is a random walk on $\mathbb{Z}^{2}$. It follows from the invariance principle for random walks that the law of $J_{(i i)} n^{\alpha+\gamma-1} m^{-1 / 2}$ converges as $n \rightarrow \infty$ to the exit time for a standard two dimensional Brownian motion from the unit disk, having started at the origin. In particular this distribution does not put mass at the value 0 and does not depend on $m$. It is thus possible to fix $m$ large enough such that

$$
\mathbb{P}\left[J_{(i i)} n^{\alpha+\gamma-1} \geq k \mid \tau\right] \geq 1-\delta / 4
$$

From the same reason we can choose $K>k$ such that

$$
\mathbb{P}\left[J_{(i i)} n^{\alpha+\gamma-1} \leq K \mid \boldsymbol{\tau}\right] \geq 1-\delta / 4 .
$$

Hence, outside a set of probability $\delta / 2$ the number of parts before $J_{(i i)}$ is in interval $\left(k n^{1-\alpha-\gamma}, K n^{1-\alpha-\gamma}\right)$. We use $A$ to denote this event.

Conditionally on $A$, we will show that

$$
\mathbb{P}\left[\min \left(J_{(i)}, J_{(i i i)}, J_{(i v)}, J_{(v)}\right) \leq J_{(i i)} \mid \tau, A\right] \rightarrow 0 \quad \text { as } \quad n \rightarrow \infty
$$


The claim of the lemma is then an easy consequence of this fact and the previous paragraph. Observe that (107) means that in the majority of cases the first bad event that happens is the possibility of exit from $\mathbb{D}$. The probability of all other events is negligible.

We start with condition (iv). According to it, the part is bad if its end is not in $\mathcal{E}(n)$. Lemma 5.8 states that the probability that this happens during a particular part of the trajectory is of order $n^{2-\kappa / 2-\gamma / 2}$. Since the number of parts before $J_{(i i)}$ is bounded by $K n^{1-\alpha-\gamma}$, the probability that (iv) happens is bounded by $K n^{3-\alpha-\gamma / 2-\kappa / 2}$. However, $\kappa$ can be chosen large enough to assure that this bound converges to 0 . We thus have

$$
\mathbb{P}\left[J_{(i v)}<J_{(i i)} \mid \boldsymbol{\tau}, A\right] \rightarrow 0 .
$$

Using a very similar reasoning and Lemma 5.10 we get exactly the same estimate for condition (v). Hence,

$$
\mathbb{P}\left[J_{(v)}<J_{(i i)} \mid \tau, A\right] \rightarrow 0
$$

Condition (i) requires that $X$ does not visit two deep traps during one part of the trajectory. We use $B$ to denote the event $A \cap\left\{J_{(i v)}>J_{(i i)}\right\}$. We show

$$
\mathbb{P}\left[J_{(i)}<J_{(i i)} \mid B, \tau\right] \rightarrow 0 .
$$

Since we assume that $J_{(i v)} \geq J_{(i i)}$, we can apply Lemma 5.7. It claims that probability of hitting two deep traps during one part is of order $n^{2(\alpha+\gamma-1)}$. By the same argument as before we can bound the probability in (110) by $K n^{\alpha+\gamma-1}$ and it tends to 0 as $n \rightarrow \infty$.

The last condition (iii) demands that $X$ does not return to a deep trap after exiting the disk of area $2^{n} n^{-\kappa}$ around it. For one particular trap probability of such event can be bounded by $c n^{-1} \log n$ by Lemma 5.11. According to Lemma 5.2, the probability of visiting a deep trap during one part of the trajectory is of order $n^{\alpha+\gamma-1}$. Let $N$ denotes the number of visited deep traps before $\Lambda$. Conditionally on $B$, it is not difficult to show using Markov inequality that

$$
\mathbb{P}\left[N \geq n^{1 / 2} \mid B, \boldsymbol{\tau}\right] \leq C n^{-1 / 2} .
$$

We have thus

$$
\begin{aligned}
& \mathbb{P}\left[J_{(i i i)}<J_{(i i)} \mid B, \boldsymbol{\tau}\right] \\
& \quad \leq \mathbb{P}\left[J_{(i i i)}<J_{(i i)} \mid B, \boldsymbol{\tau}, N \leq n^{1 / 2}\right] \mathbb{P}\left[N \leq n^{1 / 2} \mid B, \boldsymbol{\tau}\right]+\mathbb{P}\left[N \geq n^{1 / 2} \mid B, \boldsymbol{\tau}\right] \\
& \quad \leq c n^{-1 / 2} \log n+C n^{-1 / 2} \rightarrow 0 \quad \text { as } n \rightarrow \infty
\end{aligned}
$$

The claim (107) that follows easily from (108)-(112). This finishes the proof of Lemma 5.1. 


\section{Properties of the score}

In this section we will prove the convergence of the sequence of processes $Y^{n}$ to a Lévy process. This result is contained in Proposition 6.5. Recall that $Y^{n}$ was defined in (27) as a properly rescaled sum of scores. Hence, we should first study the properties of the score.

The score of the $i$-th part of the trajectory depends on the history only through its starting point $x_{i}^{n}$. We thus associate to every point $x \in \mathcal{E}(n)$ the random variable $s_{x}$, which has the same distribution as the score of a part of the trajectory of $X$ that is started at $x$. We can ignore the points in $\mathbb{D}(n) \backslash \mathcal{E}(n)$ because we do not consider the parts of trajectory started in this set (see definition of $J$ ). We have got already some information which can help us to describe the distribution of the random variables $s_{x}$. According to Lemma 5.7, the probability of hitting two deep traps in the disk $D_{x}\left(2^{n} n^{\gamma}\right)$ is of order $n^{2(\alpha+\gamma-1)}$, and the probability of hitting one deep trap is with high precision $\mathcal{K} p_{\varepsilon}^{M} n^{\alpha+\gamma-1}$. Otherwise $X$ does not hit any deep trap. In the last case $s_{x}=0$ (if none of (i)-(v) of Section 2 happen).

We want now to study more precisely the distribution of $s_{x}$ conditionally on $s_{x}<\infty$. To achieve it we should gain more information about the depth of the trap that $X$ hits as the first. The idea behind the proof is that as $n$ increases the density of deep traps becomes lower, and the hitting measure of $T_{\varepsilon}^{M}$ charges more and more sites. The distribution of the depth of the first visited trap should be thus close to the original distribution of the depth of the trap conditioned on being between $\varepsilon 2^{n / \alpha} / n$ and $M 2^{n / \alpha} / n$.

To prove this heuristics we divide the set of deep traps into several parts and we estimate the probability of hitting each of them. Let $h(x)$ be a function satisfying

$$
h(x) \geq(\log x)^{-1}, \quad \lim _{x \rightarrow \infty} h(x)=0,
$$

and (with $L$ defined in (16))

$$
L\left(2^{n / \alpha} n^{-1} x\right)-1=o(h(n)) \text { for all } x \geq \varepsilon .
$$

Such function exists because $\lim _{x \rightarrow \infty} L(x)=1$. Let $z_{n}(i)$ satisfy $\varepsilon=z_{n}(0)<$ $z_{n}(1)<\cdots<z_{n}(R)=M$ and $z_{n}(i+1)-z_{n}(i) \in(h(n), 2 h(n))$ for all $i \in$ $\{0, \ldots R-1\}$.

We now estimate the probability of hitting a trap in $T_{z_{n}(i)}^{z_{n}(i+1)}$. We use $p_{i}^{n}$ to denote

$$
p_{i}^{n}=z_{n}(i)^{-\alpha}-z_{n}(i+1)^{-\alpha}
$$

Lemma 6.1. For any $\delta>0$ and $\mathbb{P}$-a.e. $\tau$ there exists $n_{0}$ such that for all $n>n_{0}$, for all $x \in \mathcal{E}(n)$, and for all $i=\{0, \ldots, R-1\}$ the probability that the simple random walk started at $x$ hits a trap in $T_{z_{n}(i)}^{z_{n}(i+1)}$ before the exit from $D_{x}\left(2^{n} n^{\gamma}\right)$ is in the interval

$$
\left[\mathcal{K}(1-\delta) n^{\alpha+\gamma-1} p_{i}^{n}, \mathcal{K}(1+\delta) n^{\alpha+\gamma-1} p_{i}^{n}\right]
$$


Proof. The proof is very similar to the proof of Lemma 5.2. We should first improve the bounds on the homogeneity of the environment that we have proved in Lemma 5.3 .

Let $H_{6}=H_{6}(n, \delta, \varepsilon, M)$ be the event that for every square $B_{x}\left(2^{n} n^{v}\right)$ in $\mathbb{D}(n)$ and for every $i \in\{0, \ldots R-1\}$ the number of sites in $T_{z_{n}(i)}^{z_{n}(i+1)} \cap B_{x}\left(2^{n} n^{\nu}\right)$ is in the interval

$$
\left[(1-\delta) n^{\alpha+v} p_{i}^{n},(1+\delta) n^{\alpha+v} p_{i}^{n}\right]
$$

We prove that $H_{6}$ occurs $\mathbb{P}$-a.s. for $n$ large enough.

Lemma 6.2. For any $\delta$ there exist constants $c$ and $C$ such that for $n$ large enough

$$
\mathbb{P}\left[H_{6}\right] \geq 1-C \log (n) n^{1-\alpha-v} \delta^{-2} \exp \left(-c n^{\nu+\alpha} h(n)\right) .
$$

Using this lemma it is not difficult to finish the proof of Lemma 6.1. We will not give the detailed reasoning, because the proof follows the same line as the proof of Lemma 5.2. The only change is that Lemma 6.2 should be used instead of Lemma 5.3.

Proof of Lemma 6.2. To show that $H_{6}$ occurs $\mathbb{P}$-a.s. for $n$ large enough we will need the following technical lemma that estimates the probability that a trap is in $T_{z_{n}(i)}^{z_{n}(i+1)}$.

Lemma 6.3. For any $\eta>0$ there exist $n_{0}$ such that for all $n \geq n_{0}$ and all $i=$ $0, \ldots, R-1$

$$
\mathbb{P}\left[0 \in T_{z_{n}(i)}^{z_{n}(i+1)}\right] \in\left((1-\eta) \frac{n^{\alpha}}{2^{n}} p_{i}^{n},(1+\eta) \frac{n^{\alpha}}{2^{n}} p_{i}^{n}\right)
$$

Proof. Let $g(x)=L(x)-1$. Then by (16) we have

$$
\begin{aligned}
\mathbb{P}\left[0 \in T_{z_{n}(i)}^{z_{n}(i+1)}\right] & =\mathbb{P}\left[\tau_{0} \in\left[z_{n}(i) \frac{2^{n / \alpha}}{n}, z_{n}(i+1) \frac{2^{n / \alpha}}{n}\right)\right] \\
= & \frac{n^{\alpha}}{2^{n}}\left[p_{i}^{n}+\frac{g\left(2^{n / \alpha} n^{-1} z_{n}(i)\right)}{z_{n}(i)^{\alpha}}-\frac{g\left(2^{n / \alpha} n^{-1} z_{n}(i+1)\right)}{z_{n}(i+1)^{\alpha}}\right] .
\end{aligned}
$$

We should thus show that

$$
\frac{g\left(2^{n / \alpha} n^{-1} z_{n}(i)\right)}{z_{n}(i)^{\alpha}}-\frac{g\left(2^{n / \alpha} n^{-1} z_{n}(i+1)\right)}{z_{n}(i+1)^{\alpha}}=o\left(p_{i}^{n}\right) .
$$

However, this is obviously true since

$$
p_{i}^{n}=\left(z_{n}(i)\right)^{-\alpha}-\left(z_{n}(i+1)\right)^{-\alpha} \geq \operatorname{ch}(n)
$$

for some $c$ depending only on $M$, and $g\left(2^{n / \alpha} n^{-1} z_{j}^{n}\right)=o(h(n))$ by (114). 
The remaining part of the proof of Lemma 6.2 is analogous to the proof of Lemma 5.3. We only explain the appearance of the additional factors $\log (n)$ and $h(n)$ that are in (118) but not in (63). The logarithm before the exponential is due to the summation over all possible values of $i$ and (113). The factor $h(n)$ inside the exponent comes from Lemma 6.3 which replaces the bound on $p$ before (66), and from the existence of constants $c(\varepsilon, M), C(\varepsilon, M)$ such that

$$
\operatorname{ch}(n) \leq \frac{1}{z_{n}(i)^{\alpha}}-\frac{1}{z_{n}(i+1)^{\alpha}} \leq C h(n) .
$$

This finishes the proof.

Using Lemma 6.1 we can now describe the behaviour of random variables $s_{x}$. Due to condition (ii) from Section 2, all good parts of the trajectory start at sites that are in the distance larger than $\sqrt{\pi^{-1} 2^{n} n^{\gamma}}$ from the border of $\mathbb{D}(n)$. That is why we introduce $\mathcal{E}_{0}(n)=\left\{x \in \mathcal{E}(n): D_{x}\left(2^{n} n^{\gamma}\right) \cap \mathbb{D}(n)^{c}=\emptyset\right\}$. The random variables $s_{x}$ then satisfy

\section{Lemma 6.4. For $\mathbb{P}$-a.e. random environment $\tau$}

$$
\begin{aligned}
& \lim _{n \rightarrow \infty} \max _{x \in \mathcal{E}_{0}(n)} \frac{1-\mathbb{E}\left[\exp \left(-\frac{\lambda s_{x}}{2^{n / \alpha}}\right) \mid s_{x}<\infty, \boldsymbol{\tau}\right]}{n^{\alpha+\gamma-1}}=F(\lambda), \\
& \lim _{n \rightarrow \infty} \min _{x \in \mathcal{E}_{0}(n)} \frac{1-\mathbb{E}\left[\exp \left(-\frac{\lambda s_{x}}{2^{n / \alpha}}\right) \mid s_{x}<\infty, \boldsymbol{\tau}\right]}{n^{\alpha+\gamma-1}}=F(\lambda),
\end{aligned}
$$

with

$$
F(\lambda)=F(\lambda ; \varepsilon, M, \alpha)=\mathcal{K}\left(p_{\varepsilon}^{M}-\int_{\varepsilon}^{M} \frac{\alpha}{1+\mathcal{K}^{\prime} \lambda z} \cdot \frac{1}{z^{\alpha+1}} d z\right)
$$

and $\mathcal{K}^{\prime}=\pi^{-1} \log 2$.

Proof. By Lemmas 5.2, 5.8, and 5.10 we know that if $\kappa$ is large enough, then $\mathbb{P}\left[s_{x}=\infty\right]=O\left(n^{2(\alpha+\gamma-1)}\right)$. Since this probability is much smaller than any other probability that will be used in the following computation, the conditioning on $s_{x}<\infty$ has almost no effect. Actually,

$$
\begin{aligned}
\mathbb{E}[\exp ( & \left.\left.-\frac{\lambda s_{x}}{2^{n / \alpha}}\right) \mid s_{x}<\infty, \boldsymbol{\tau}\right] \\
& =\mathbb{P}\left[s_{x}<\infty \mid \boldsymbol{\tau}\right]^{-1} \mathbb{E}\left[\exp \left(-\frac{\lambda s_{x}}{2^{n / \alpha}}\right) \chi\left\{s_{x}<\infty\right\} \mid \boldsymbol{\tau}\right] \\
& =\mathbb{E}\left[\exp \left(-\frac{\lambda s_{x}}{2^{n / \alpha}}\right) \mid \boldsymbol{\tau}\right]\left(1+O\left(n^{2(\alpha+\gamma-1)}\right)\right) .
\end{aligned}
$$

If the process $X$ hits deep trap $y$ in $D_{x}\left(2^{n} n^{\gamma}\right)$ and nothing unusual happens, then the random variable $s_{x}$ is a sum of a geometrically distributed number of exponential random variables with mean $\tau_{y}$. The mean of the geometrically distributed number of visits of $y$ is equal to $G_{D\left(2^{n} n^{-\kappa}\right)}(0,0)$, where by (227)

$$
G_{D\left(2^{n} n^{-\kappa}\right)}(0,0)=\frac{2}{\pi} \log \sqrt{\pi^{-1} 2^{n} n^{-\kappa}}+O(1)=\frac{n}{\pi} \log 2+O(\log n) .
$$


Since the geometrically long sum of exponential random variables is again exponentially distributed, the score $s_{x}$ is in this case an exponential random variable with mean $\tau_{y}(n \log 2 / \pi+O(\log n))$. This implies that conditionally on hitting a trap with the depth $\tau_{y}$ the Laplace transform of $s_{x} / 2^{n / \alpha}$ equals

$$
\mathbb{E}\left[\exp \left(-\frac{\lambda s_{x}}{2^{n / \alpha}}\right) \mid \tau_{y}\right]=\frac{1}{1+\lambda \tau_{y} 2^{-n / \alpha}(n \log 2 / \pi+O(\log n))} .
$$

We now estimate the Laplace transform $\mathbb{E}\left[\exp \left(-\lambda s_{x} 2^{-n / \alpha}\right) \mid \tau\right]$. We start with a lower bound. Choose $\delta>0$. By Lemmas 5.7, 6.1, and expression (128) we have for $n$ large enough

$$
\begin{aligned}
& \mathbb{E}\left[\exp \left(-\frac{\lambda s_{x}}{2^{n / \alpha}}\right) \mid \boldsymbol{\tau}\right] \geq\left(1-(1+\delta) \mathcal{K} p_{\varepsilon}^{M} n^{\alpha+\gamma-1}\right) \\
& \quad+\mathcal{K} n^{\alpha+\gamma-1} \sum_{i=1}^{R} \frac{1-\delta}{1+\lambda \frac{z_{n}(i)}{2^{n / \alpha}} \frac{2^{n / \alpha}}{n} \frac{n}{\pi} \log 2+o(1)}\left(\frac{1}{\left(z_{i-1}^{n}\right)^{\alpha}}-\frac{1}{\left(z_{n}(i)\right)^{\alpha}}\right)
\end{aligned}
$$

The last expression can be bounded from below by

$$
1-\mathcal{K} n^{\alpha+\gamma-1}\left(p_{\varepsilon}^{M}-\int_{\varepsilon}^{M} \frac{\alpha}{1+\mathcal{K}^{\prime} \lambda z} \frac{1}{z^{\alpha+1}} d z\right)-\delta C n^{\alpha+\gamma-1} p_{\varepsilon}^{M},
$$

with $C$ being a constant not depending on $\delta$. The last expression together with (126) give

$$
\begin{aligned}
& \limsup _{n \rightarrow \infty} \max _{x \in \mathcal{E}_{0}(n)} \frac{1-\mathbb{E}\left[\exp \left(-\frac{\lambda s_{x}}{2^{n / \alpha}}\right) \mid s_{x}<\infty, \boldsymbol{\tau}\right]}{n^{\alpha+\gamma-1}} \\
& \quad \leq \mathcal{K}\left(p_{\varepsilon}^{M}-\int_{\varepsilon}^{M} \frac{\alpha}{1+\mathcal{K}^{\prime} \lambda z} \frac{1}{z^{\alpha+1}} d z\right)+C \delta p_{\varepsilon}^{M} .
\end{aligned}
$$

Since $\delta$ can be taken arbitrarily small, the proof of the upper bound for the first expression in (124) is finished. The proof of the lower bound for the second expression in (124) is completely similar.

We can finally show the convergence of the sequence $Y^{n}$ to a Lévy process (see [Ber96] for complete treatment of Lévy processes). The following proposition will be used later to prove aging.

Proposition 6.5. For $\mathbb{P}$-a.e. realisation of the environment, the sequence of processes $Y^{n}(t)$ converges weakly in the Skorokhod topology on $D([0, \infty))$ to the Lévy process $Y(t)$ with the Lévy measure

$$
\rho(d x)=\frac{\alpha \mathcal{K}}{\mathcal{K}^{\prime}} \int_{\varepsilon}^{M} \frac{1}{z^{\alpha+2}} \exp \left(-\frac{x}{\mathcal{K}^{\prime} z}\right) d z d x
$$


Proof. We first prove the weak convergence of finite dimensional distributions. Let $0=t_{0}<t_{1}<\cdots<t_{\ell}$. We will show the convergence of Laplace transforms. By definition of $Y^{n}$

$$
\mathbb{E}\left[\exp \left(-\sum_{i=1}^{\ell} \lambda_{i}\left(Y^{n}\left(t_{i}\right)-Y^{n}\left(t_{i-1}\right)\right)\right)\right]=\mathbb{E}\left[\prod_{i=1}^{\ell} \prod_{j \in B(n, i)} \exp \left(-\frac{\lambda_{i}}{2^{n / \alpha}} s_{j}^{n}\right)\right],
$$

where $B(n, i)=\left\{\left\lfloor n^{1-\alpha-\gamma} t_{i-1}\right\rfloor+1, \ldots,\left\lfloor n^{1-\alpha-\gamma} t_{i}\right\rfloor\right\}$.

If $j<J$, then the random variables $s_{j}^{n}$ are determined by behaviour of $X$, otherwise they are equal to $\tilde{s}_{j}^{n}$. Since $\tilde{s}_{j}^{n}$ 's are independent of all other randomness, we can write

$$
\begin{aligned}
= & \sum_{k=0}^{\infty} \mathbb{P}[J=k] \mathbb{E}\left[\prod_{i=1}^{\ell} \prod_{\substack{j \in B(n, i) \\
j<k}} \exp \left(-\frac{\lambda_{i}}{2^{n / \alpha}} s_{j}^{n}\right) \mid J=k\right] \\
& \mathbb{E}\left[\prod_{i=1}^{\ell} \prod_{\substack{j \in B(n, i) \\
j \geq k}} \exp \left(-\frac{\lambda_{i}}{2^{n / \alpha}} \tilde{s}_{j}^{n}\right) \mid J=k\right] .
\end{aligned}
$$

At this place it is necessary to define the distribution of $\tilde{s}_{j}^{n}$. We require that $\tilde{s}_{i}^{n}$ 's satisfy the same relation as $s_{x}$ in the limit, i.e.

$$
\mathbb{E}\left[\exp \left(-\frac{\lambda}{2^{n / \alpha}} \tilde{s}_{j}^{n}\right)\right]=1-F(\lambda) n^{\alpha+\gamma-1} .
$$

We have obviously chosen the $\tilde{s}_{j}^{n}$ 's in the way that the second expectation in (134) does not pose any problems. We should thus control only the first one.

Let $\boldsymbol{y}=\left\{y_{0}, \ldots, y_{k}\right\} \in \mathcal{E}(n)^{k+1}$. We use $\boldsymbol{x}_{n}$ to denote the sequence $x_{0}^{n}, \ldots, x_{k}^{n}$ of starting points of the parts of the trajectory. We have

$$
\begin{aligned}
& \mathbb{E}\left[\prod_{i=1}^{\ell} \prod_{\substack{j \in B(n, i) \\
j<k}} \exp \left(-\frac{\lambda_{i}}{2^{n / \alpha}} s_{j}^{n}\right)\right] \\
& \quad=\sum_{\boldsymbol{y}} \mathbb{P}\left[\boldsymbol{x}_{n}=\boldsymbol{y}\right] \mathbb{E}\left[\left.\prod_{i=1}^{\ell} \prod_{\substack{j \in B(n, i) \\
j<k}} \exp \left(-\frac{\lambda_{i}}{2^{n / \alpha}} s_{j}^{n}\right)\right|_{n}=\boldsymbol{y}\right] .
\end{aligned}
$$

Only the last term of the product depends on $y_{k}$. We can thus sum over all possible values of the endpoint of the last part. Let $\boldsymbol{x}{ }_{n}^{\prime}$, resp. $\boldsymbol{y}^{\prime}$, denote the sequences $\boldsymbol{x}_{n}$ and $\boldsymbol{y}$ without the last element. We get

$$
=\sum_{\boldsymbol{y}^{\prime}} \mathbb{P}\left[\boldsymbol{x}_{n}^{\prime}=\boldsymbol{y}^{\prime}\right] \mathbb{E}\left[\prod_{i=1}^{\ell} \prod_{\substack{j \in B(n, i) \\ j<k}} \exp \left(-\frac{\lambda_{i}}{2^{n / \alpha}} s_{j}^{n}\right) \mid \boldsymbol{x}_{n}^{\prime}=\boldsymbol{y}^{\prime}\right] .
$$


Conditionally on the value $x_{k-1}^{n}$, the random variable $s_{k-1}^{n}$ is independent of the rest. The expectation in the last formula can be thus written as

$$
\mathbb{E}\left[\prod_{i=1}^{\ell} \prod_{\substack{j \in B(n, i) \\ j<k-1}} \exp \left(-\frac{\lambda_{i}}{2^{n / \alpha}} s_{j}^{n}\right) \mid \boldsymbol{x}_{n}^{\prime}=\boldsymbol{y}^{\prime}\right] \mathbb{E}\left[\exp \left(-\frac{\lambda_{r}}{2^{n / \alpha}} s_{x_{k-1}^{n}}\right) \mid s_{x_{k-1}^{n}}<\infty\right],
$$

where the index $r$ satisfies $k-1 \in B(n, r)$. According to Lemma 6.4, the second expectation can be bounded from above by

$$
1-(1-\delta) F\left(\lambda_{r}\right) n^{\alpha+\gamma-1}
$$

if $n$ is large enough.

We can now repeat the same manipulation with the last but one value of $j$, etc. At the end, putting the result of this iteration into (134), we get

$$
\begin{aligned}
& \mathbb{E}\left[\exp \left(-\sum_{i=1}^{\ell} \lambda_{i}\left(Y^{n}\left(t_{i}\right)-Y^{n}\left(t_{i-1}\right)\right)\right)\right] \\
& \quad \leq \prod_{i=1}^{\ell}\left(1-(1-\delta) F\left(\lambda_{i}\right) n^{\alpha+\gamma-1}\right)^{\left\lfloor n^{1-\alpha-\gamma}\left(t_{i}-t_{i-1}\right)\right\rfloor} .
\end{aligned}
$$

Taking the limits we obtain

$$
\begin{aligned}
& \underset{n \rightarrow \infty}{\limsup } \mathbb{E}\left[\exp \left(-\sum_{i=1}^{\ell} \lambda_{i}\left(Y^{n}\left(t_{i}\right)-Y^{n}\left(t_{i-1}\right)\right)\right)\right] \\
& \leq \exp \left[-\sum_{i=1}^{\ell}(1-\delta) F\left(\lambda_{i}\right)\left(t_{i}-t_{i-1}\right)\right] .
\end{aligned}
$$

In the same way we obtain a lower bound. Since $\delta$ was arbitrary we have

$$
\begin{aligned}
& \lim _{n \rightarrow \infty} \mathbb{E}\left[\exp \left(-\sum_{i=1}^{\ell} \lambda_{i}\left(Y^{n}\left(t_{i}\right)-Y^{n}\left(t_{i-1}\right)\right)\right)\right] \\
& \quad=\exp \left[-\sum_{i=1}^{\ell} F\left(\lambda_{i}\right)\left(t_{i}-t_{i-1}\right)\right] .
\end{aligned}
$$

The corresponding Laplace transform of $Y(t)$ is easy to calculate. We have

$$
\mathbb{E}\left[\exp \left(-\sum_{i=1}^{\ell} \lambda_{i}\left(Y\left(t_{i}\right)-Y\left(t_{i-1}\right)\right)\right)\right]=\exp \left[-\sum_{i=1}^{\ell} \Psi\left(\lambda_{i}\right)\left(t_{i}-t_{i-1}\right)\right],
$$

where $\Psi(\lambda)$ is the Laplace exponent of $Y$. By Lévy-Khintchine formula it is equal to

$$
\Psi(\lambda)=\int_{0}^{\infty}\left(1-e^{-\lambda x}\right) \rho(d x)
$$


An easy integration gives the same result as (142).

To prove the weak convergence it remains to verify that the sequence $Y_{n}$ is tight. We use Theorem 16.8 from [Bil99]. We should show that for any $N$ and $\delta_{1}, \delta_{2}$ there exist $a, n_{0}$, and $\eta$ such that

$$
\begin{array}{ll}
\mathbb{P}\left[\sup _{t \in[0, N]}\left|Y_{n}(t)\right| \geq a\right]<\delta_{1} & \text { for all } n>n_{0} \\
\mathbb{P}\left[w\left(Y^{n}, \eta, N\right) \geq \delta_{2}\right]<\delta_{1} & \text { for all } n>n_{0},
\end{array}
$$

where

$$
w(f, \eta, N)=\inf _{\left\{t_{i}\right\}} \max _{0<i \leq r} \sup \left\{|f(s)-f(t)|: s, t \in\left[t_{i-1}, t_{i}\right)\right\}
$$

and the infimum runs over all finite collections $\left\{t_{i}\right\}$ such that $0<t_{i}-t_{i-1}<\eta$, $t_{0}=0$, and $t_{r}=N$.

Proof of (i) Since $Y^{n}$ are increasing, (i) is equivalent to the tightness of the sequence $Y^{n}(N)$. From convergence of finite dimensional distribution we know that the Laplace transforms of $Y^{n}(N)$ converge to $\mathcal{L}_{Y(N)}(\lambda)=\mathbb{E}[\exp (-\lambda Y(N))]$. It is sufficient to verify that this Laplace transform satisfies $\lim _{\lambda \rightarrow 0} \mathcal{L}_{Y(N)}(\lambda)=1$. However, $\mathcal{L}_{Y(N)}$ is continuous and

$$
\mathcal{L}_{Y(N)}(0)=\exp (-N F(0))=\exp \left[-N \mathcal{K}\left(p_{\varepsilon}^{M}-\int_{\varepsilon}^{M} \frac{\alpha}{z^{\alpha+1}} d z\right)\right]=1 .
$$

Proof of (ii) According to Lemma 5.7, the expected number of jumps of $Y^{n}$ in the interval $[0, N]$ can be bounded by some constant $C$ not depending on $n$. Markov inequality then gives the existence of some $C^{\prime}$ such that the probability that the number of jumps of $Y^{n}$ exceeds $C^{\prime}$ is smaller than $\delta_{1} / 2$ for all $n$ large enough. If the number of jumps is finite, we can take $\left\{t_{i}\right\}$ being the superset of the set of all jumps. The process $Y^{n}$ is then constant on any interval $\left[t_{i-1}, t_{i}\right)$ and thus $w\left(Y^{n}, \eta, N\right)=0$. This completes the proof of Proposition 6.5.

\section{Proof of aging}

We prove here the following proposition that is a more precise version of Theorem 1.1 .

Proposition 7.1. For $\mathbb{P}$-a.e. realisation of the environment $\boldsymbol{\tau}$ and for every $0<$ $\theta<\infty$

$$
\lim _{t \rightarrow \infty} R(t, t+\theta t)=\int_{0}^{1 / 1+\theta} \frac{\sin \alpha \pi}{\pi} u^{\alpha-1}(1-u)^{-\alpha} d u \equiv R(\theta) .
$$

An easy calculation gives

Corollary 7.2. The function $R(\theta)$ satisfies

$$
\lim _{\theta \rightarrow 0} R(\theta)=1 \quad \text { and } \quad \lim _{\theta \rightarrow \infty} R(\theta)=0 .
$$


Proof. I. We introduce some additional notation. Let $Z(t)=Z(t ; \varepsilon, M)$ be a Lévy process with the Lévy measure

$$
\rho^{\prime}(d x)=\frac{\alpha \mathcal{K}}{\mathcal{K}^{\prime}}\left(\int_{0}^{\varepsilon}+\int_{M}^{\infty}\right) \frac{1}{z^{\alpha+2}} \exp \left(-\frac{x}{\mathcal{K}^{\prime} z}\right) d z d x,
$$

independent of the processes $Y, Y^{n}$. We define the new family of processes,

$$
\tilde{Y}^{n}(t)=Y^{n}(t)+Z(t) \quad \text { and } \quad \tilde{Y}(t)=Y(t)+Z(t) .
$$

The advantage of this new class is that the Lévy measure of $\tilde{Y}$ is given by

$$
\begin{aligned}
\rho(d x)+\rho^{\prime}(d x) & =\frac{\alpha \mathcal{K}}{\mathcal{K}^{\prime}} \int_{0}^{\infty} \frac{1}{z^{\alpha+2}} \exp \left(-\frac{x}{\mathcal{K}^{\prime} z}\right) d z d x \\
& =\frac{\alpha^{2} \Gamma(\alpha) \mathcal{K}\left(\mathcal{K}^{\prime}\right)^{\alpha}}{x^{\alpha+1}} d x,
\end{aligned}
$$

and thus $\tilde{Y}$ is an $\alpha$-stable subordinator. As an easy consequence of the previous section we know that the sequence $\tilde{Y}^{n}$ converges weakly to $\tilde{Y}$ whatever the values chosen for $\varepsilon$ and $M$. Let $\mathcal{R}_{n}=\mathcal{R}\left(\tilde{Y}^{n}\right), \mathcal{R}=\mathcal{R}(\tilde{Y})$ denote the range of $\tilde{Y}^{n}$, resp. of $\tilde{Y}$.

Fix $\theta>0$. Let $\delta_{1}, \delta_{2}>0$ be arbitrarily small but fixed. We will now fix the values of $M, m, \varepsilon$ as functions of $\delta_{1}, \delta_{2}$ and $n$ as a function of $\delta_{1}, \delta_{2}$ and $t$. First, let $n(t)$ be the integer satisfying

$$
1 \leq \frac{t}{2^{n(t) / \alpha}}<2^{1 / \alpha}
$$

Obviously, $n(t) \rightarrow \infty$ as $t \rightarrow \infty$. In this section $n=n(t)$ is always connected with $t$ via (152). We use $s=s(t)$ to denote the rescaled value of $t, s=t 2^{-n(t) / \alpha}$. By (152) $s$ satisfies $1 \leq s<2^{1 / \alpha}$. In the same way we rescale the value $(1+\theta) t$. The process $\tilde{Y}^{n}$ that we will use to approximate the time change $\bar{S}^{n}$ should be thus relevant until the level $(1+\theta) s<(1+\theta) 2^{1 / \alpha}$. Let $t_{0}$ be such that

$$
\mathbb{P}\left[\tilde{Y}\left(t_{0}\right)<(1+\theta) 2^{1 / \alpha}\right]<\delta_{1} .
$$

By the weak convergence of $\tilde{Y}^{n}$ to $\tilde{Y}$ we can take $t$ (and so $n=n(t)$ ) large enough such that

$$
\mathbb{P}\left[\tilde{Y}^{n(t)}\left(t_{0}\right) \geq(1+\theta) 2^{1 / \alpha}\right]>1-2 \delta_{1} .
$$

There are $J(n)$ relevant parts of the trajectory of the process $X$. For every time unit we need $n^{1-\alpha-\gamma}$ parts. So, we should choose $m$ in such a way that

$$
\mathbb{P}\left[J(n) n^{\alpha+\gamma-1} \geq t_{0}\right]>1-\delta_{1} .
$$

By Lemma 5.1, this can be done independently of $\varepsilon$ and $M$. Let $A_{1}$ be the event $\left\{\tilde{Y}^{n}\left(t_{0}\right) \geq(1+\theta) s\right.$ and $\left.J(n) \geq t_{0} n^{1-\alpha-\gamma}\right\}$. Then, by (154) and (155),

$$
\mathbb{P}\left[A_{1}\right] \geq 1-3 \delta_{1} \text {. }
$$


We can now fix the values of $\varepsilon$ and $M$. Later, we want to work with the processes $\tilde{Y}^{n}$ instead of $Y^{n}$. We should thus guarantee that the artificial addition of process $Z$ is not relevant. We take $\varepsilon_{1}$ and $M_{1}$, such that

$$
\mathbb{P}\left[Z\left(t_{0} ; \varepsilon_{1}, M_{1}\right)>\delta_{2}\right]<\delta_{1} .
$$

We can also safely ignore the error introduced by the very deep and the shallow traps. Indeed, by Lemma 4.1 , we can take $M_{2}$ such that

$$
\mathbb{P}\left[X_{d} \text { hits } T_{M_{2}} \text { before } \Lambda_{d}(n)\right]<\delta_{1} .
$$

Further, by Lemma 3.1, we know that there is a constant $K_{1}$ (not depending on $\varepsilon, M$ or $n$ ), such that $\tau$-a.s. for $n$ (or equivalently $t$ ) large enough

$$
\mathbb{E}\left[\frac{1}{2^{n / \alpha}} \cdot \text { time spent in } T^{\varepsilon} \text { before } \Lambda_{d}(n) \mid \boldsymbol{\tau}\right] \leq K_{1} \varepsilon^{1-\alpha},
$$

and thus

$$
\mathbb{P}\left[\frac{1}{2^{n / \alpha}} \cdot \text { time spent in } T^{\varepsilon} \text { before } \Lambda_{d}(n)>\delta_{2} \mid \tau\right] \leq \delta_{2}^{-1} \varepsilon^{1-\alpha} K_{1} .
$$

Let us take $\varepsilon_{2}$ such that $\delta_{2}^{-1} \varepsilon_{2}^{1-\alpha} K_{1}<\delta_{1}$. The constants $\varepsilon$ and $M$ are then defined by

$$
\varepsilon=\min \left(\varepsilon_{1}, \varepsilon_{2}\right) \quad \text { and } \quad M=\max \left(M_{1}, M_{2}\right) .
$$

This choice of constants ensures that the distance between the rescaled time change $\bar{S}^{n}$ and the process $\tilde{Y}^{n}$ is small. Precisely, let

$$
A_{2}=\left\{\left|\bar{S}^{n}(t)-\tilde{Y}^{n}(t)\right| \leq 2 \delta_{2} \forall t \leq t_{0}\right\} .
$$

Then our choice of constants gives

$$
\mathbb{P}\left[A_{2} \mid A_{1}\right] \geq 1-3 \delta_{1} \text {. }
$$

Let $A=A_{1} \cap A_{2}$. Then from (156) and (163) follows that for $t$ large enough

$$
\mathbb{P}[A] \geq 1-6 \delta_{1} .
$$

II. Later we will take the limit $n \rightarrow \infty$ for fixed value of $s \in\left[1,2^{1 / \alpha}\right]$ instead of taking limit $t \rightarrow \infty$. We will show that this limit does not depend on $s$. To be able to show the existence of the limit $t \rightarrow \infty$ we will need uniformity of convergence in $s$. The proof of the following auxiliary lemma is left to the reader.

Lemma 7.3. Let $\mathcal{P}_{u}(s, Y)=\mathbb{P}[[s, s+u] \cap \mathcal{R}(Y) \neq \emptyset]$ for $Y$ being $\tilde{Y}^{n}$ or $\tilde{Y}$. Then for any $u<\theta 2^{1 / \alpha}$

$$
\lim _{n \rightarrow \infty} \mathcal{P}_{u}\left(s, \tilde{Y}^{n}\right)=\mathcal{P}_{u}(s, \tilde{Y})
$$

uniformly for $s \in\left[1, \theta 2^{1 / \alpha}\right]$. 
III. We now study the event $G(t)=\{X(t)=X((1+\theta) t)\}$ for $t$ large. We divide the probability space into three disjoint parts,

$$
\begin{aligned}
E_{1}(n, s)= & \left\{\operatorname{dist}\left(s, \mathcal{R}_{n}\right) \leq 2 \delta_{2} \text { or } \operatorname{dist}\left((1+\theta) s, \mathcal{R}_{n}\right) \leq 2 \delta_{2}\right\} \\
E_{2}(n, s)= & \left\{\operatorname{dist}\left(s, \mathcal{R}_{n}\right)>2 \delta_{2}, \operatorname{dist}\left((1+\theta) s, \mathcal{R}_{n}\right)>2 \delta_{2}\right. \text { and } \\
& \left.(s,(1+\theta) s) \cap \mathcal{R}_{n} \neq \emptyset\right\} \\
E_{3}(n, s)= & \left\{\left[s-2 \delta_{2},(1+\theta) s+2 \delta_{2}\right] \cap \mathcal{R}_{n}=\emptyset\right\} .
\end{aligned}
$$

This division has the following reason. On event $A_{2}$ and therefore on event $A$, to precision $2 \delta_{2}$, any interval that does not intersect $\mathcal{R}_{n}$ corresponds to a time period that $X$ spent in $D_{y}\left(2^{n} n^{-\kappa}\right)$ around some deep trap $y$. Heuristically the points of the range correspond to times when the walk did not meet any deep trap for a long time.

We wish to show that essentially event $G(t)$ is the same as event $E_{3}(n, s)$. Obviously

$$
\begin{aligned}
\mathbb{P}\left[G(t) \cap E_{3}(n, s)\right] & \leq \mathbb{P}[G(t)] \\
& \leq \mathbb{P}\left[E_{3}(n, s)\right]+\mathbb{P}\left[E_{1}(n, s)\right]+\mathbb{P}\left[G(t) \cap E_{2}(n, s)\right]
\end{aligned}
$$

We should thus estimate all quantities in the last display. When $E_{1}$ occurs, at least one of the values $s,(1+\theta) s$ is too close to $\mathcal{R}_{n}$. Hence, we cannot know precisely what happens with the process $X$ in this situation. However, the probability of $E_{1}$ is small. Indeed,

$$
\mathbb{P}\left[E_{1}\right] \leq \mathbb{P}\left[\operatorname{dist}\left(s, \mathcal{R}_{n}\right) \leq 2 \delta_{2}\right]+\mathbb{P}\left[\operatorname{dist}\left((1+\theta) s, \mathcal{R}_{n}\right) \leq 2 \delta_{2}\right] .
$$

If $n$ is large, we can bound the first term in the last expression by

$$
\mathbb{P}\left[\operatorname{dist}\left(s, \mathcal{R}_{n}\right) \leq 2 \delta_{2}\right] \leq \delta_{1}+1-\mathbb{P}\left[\mathcal{R} \cap\left[s-2 \delta_{2}, s+2 \delta_{2}\right]=\emptyset\right] .
$$

The constant $\delta_{1}$ comes from the approximation of $\mathcal{R}_{n}$ by $\mathcal{R}$ and by Lemma 7.3 can be chosen independent of $s$. Since $\tilde{Y}$ is a stable subordinator, the probability $\mathbb{P}\left[\mathcal{R} \cap\left[s-2 \delta_{2}, s+2 \delta_{2}\right]=\emptyset\right]$ can be evaluated using formulas from Lemma B.1,

$$
\begin{aligned}
& \mathbb{P}\left[\operatorname{dist}\left(s, \mathcal{R}_{n}\right) \leq 2 \delta_{2}\right] \leq \delta_{1}+1-\mathbb{P}\left[g\left(s+2 \delta_{2}\right)<s-2 \delta_{2}\right] \\
& \quad=\delta_{1}+1-\int_{0}^{\frac{s-2 \delta_{2}}{s+2 \delta_{2}}} \frac{\sin \alpha \pi}{\pi} u^{\alpha-1}(1-u)^{-\alpha} d u \leq C \delta_{1}+C^{\prime} \delta_{2}^{1-\alpha}
\end{aligned}
$$

for some constants $C, C^{\prime}$ independent of $s$. In the same way we can estimate the second probability from (168). We have thus

$$
\mathbb{P}\left[E_{1}\right] \leq C \delta_{1}+C^{\prime} \delta_{2}^{1-\alpha} .
$$

If $A$ occurs, then the realisation of $E_{2}$ means that $X(t)$ is in disk $D_{y_{1}}\left(2^{n} n^{-\kappa}\right)$ and $X((1+\theta) t)$ is in $D_{y_{2}}\left(2^{n} n^{-\kappa}\right)$ for some $y_{1}, y_{2} \in T_{\varepsilon}^{M}$. By definition of $J$ we have necessarily $y_{1} \neq y_{2}$, and thus by Lemma 5.6

$$
\mathbb{P}\left[G(t) \cap E_{2}(n, s) \cap A\right]=0 .
$$


Hence,

$$
\mathbb{P}\left[G(t) \cap E_{2}(n, s)\right] \leq 1-\mathbb{P}[A] \leq C \delta_{1}
$$

The most interesting event is $E_{3}$. The probability of $E_{3}$ can be calculated in a similar manner to the probability of $E_{1}$. For $n$ large enough

$$
\left|\mathbb{P}\left[E_{3}(n, s)\right]-\mathbb{P}\left[\mathcal{R} \cap\left[s-2 \delta_{2},(1+\theta) s+2 \delta_{2}\right]=\emptyset\right]\right| \leq \delta_{1},
$$

which implies

$$
\left|\mathbb{P}\left[E_{3}(n, s)\right]-\int_{0}^{1 / 1+\theta} \frac{\sin \alpha \pi}{\pi} u^{\alpha-1}(1-u)^{-\alpha} d u\right| \leq\left(C \delta_{1}+C^{\prime} \delta_{2}\right) .
$$

The constants $C$ and $C^{\prime}$ can be chosen again independent of $s$. Note also that the main term does not depend on $s$.

We will now show that $\mathbb{P}\left[G(t) \cap E_{3}(n, s)\right]$ is close to $\mathbb{P}\left[E_{3}(n, s)\right]$. Let $G^{\prime}=$ $G^{\prime}(t, \theta)$ be the event that for some $y \in T_{\varepsilon}^{M}$,

$$
t^{n} \equiv \inf \{u: X(u)=y\}<t
$$

and

$$
s^{n} \equiv \sup \left\{u<\inf \left\{v>t^{n}: X(v) \notin D_{y}\left(2^{n} n^{-\kappa}\right)\right\}: X(u)=y\right\}>t(1+\theta) .
$$

Obviously we have that the event $\left\{E_{3}(n, s)\right\} \backslash\left\{G(t) \cap E_{3}(n, s)\right\}$ is contained in the event $A^{c} \cup\left(G^{\prime} \cap(\{X(t) \neq y\} \cup\{X(t(1+\theta)) \neq y\})\right.$.

Lemma 7.4. The probability of the event $G^{\prime}$ intersected with $\{X(t) \neq y\} \cup\{X(t(1+$ $\theta)) \neq y\}$ tends to zero as $t$ tends to infinity.

We use this lemma to finish the proof of Proposition 7.1. For $t$ large enough we have putting (171) and (173), into (167) we get

$$
\mathbb{P}[G(t)] \leq C \delta_{1}+C^{\prime} \delta_{2}^{1-\alpha}+\mathbb{P}\left[E_{3}(n, s)\right] .
$$

Similarly, we obtain the lower bound (for $t$ sufficiently large)

$$
\mathbb{P}[G(t)] \geq \mathbb{P}\left[E_{3}(n, s)\right]-C \delta_{1} .
$$

Since the expression (175) for $E_{3}$ and also the constants in error terms do not depend on $s$, and since $\delta_{1}$ and $\delta_{2}$ can be taken arbitrarily small, we have

$$
\lim _{t \rightarrow \infty} \mathbb{P}[G(t)]=\int_{0}^{1 / 1+\theta} \frac{\sin \alpha \pi}{\pi} u^{\alpha-1}(1-u)^{-\alpha} d u .
$$

This finishes the proof.

IV. It remains to show Lemma 7.4 
Proof of Lemma 7.4. It will suffice to show that

$$
\mathbb{P}\left[X(t)=y \mid G^{\prime}, t^{n}, y, \boldsymbol{\tau}\right], \mathbb{P}\left[X(t(1+\theta))=y \mid G^{\prime}, t^{n}, y, \boldsymbol{\tau}\right]
$$

tend to one as $t$ tends to infinity. We will only treat the first probability, the proof of the second convergence being entirely similar.

The Markov process $\left(X\left(t^{n}+s\right): s^{n}-t^{n} \geq s \geq 0\right)$, given $G^{\prime}, t^{n}, y, \boldsymbol{\tau}$ is equal in law to the process $\left(U(s): s \in\left[0, s^{n}-t^{n}\right]\right)$ conditioned on the event $\left\{S>s^{n}-t^{n}\right\}$ where $U$ and $S$ are constructed as follows:

(i) $U$ stays at site $y$ for an exponential, mean $\tau_{y}$, amount of time, then

(ii) with probability $p(n)$, the probability that a random walk starting at $y$ escapes $\mathcal{D} \equiv D_{y}\left(2^{n} n^{-\kappa}\right)$ before returning to site $y$, the process terminates and $S$ is the termination time. With probability $1-p(n)$ the process $U$ performs an excursion away from $y$ conditioned not to leave $\mathcal{D}$. At the end of the excursion it returns to $y$ and step (i) resumes and so on.

The important point is that the number $p(n)$ is of order $1 / n$ while (recall $y \in$ $T_{\varepsilon}^{M}$ ) the mean time spent at $y$ per visit exceeds $\varepsilon 2^{n / \alpha} / n$. Thus the conditioning event has probability bounded below by $C(\varepsilon, \theta)$. Hence it will suffice to show that $\mathbb{P}\left[\bar{U}\left(t-t^{n}\right) \neq y \mid \boldsymbol{\tau}\right]$ tends to zero as $t$ tends to infinity $\boldsymbol{\tau}$-a.s. where process $(\bar{U}(u): u \geq 0)$ is a Markov process that alternates staying at site $y$ an exponential amount of time with mean $\tau_{y}$ and performing excursions away from $y$ conditioned to stay within $\mathcal{D}$ (again staying at each site a time according to $\tau$ ).

We first show that $\tau$-a.s. for $t$ (and therefore $n$ ) sufficiently large, the expected duration of a conditioned excursion from $y$ is very small compared to $\tau_{y}$ uniformly over possible $y \in T_{\varepsilon}^{M}$. It is easy to prove that in the neighbourhood of $y$ there are only traps shallower than $\varepsilon n^{-5 /(1-\alpha)} 2^{n / \alpha} / n$. Indeed, as in the proof of Lemma 5.6, let

$$
B(y)=\left\{y \in T_{\varepsilon}^{M}, \exists x \in \mathcal{D}, \tau_{x} \geq \varepsilon n^{-\frac{5}{1-\alpha}} \frac{2^{n / \alpha}}{n}\right\}
$$

Then,

$$
\mathbb{P}[B(y)] \leq C 2^{n} n^{-\kappa} \frac{n^{2 \alpha} n^{\frac{5 \alpha}{1-\alpha}}}{2^{2 n}} .
$$

The summation over all sites in $\mathbb{D}(n)$ gives

$$
\mathbb{P}\left[\bigcup_{y \in \mathbb{D}(n)} B(y)\right] \leq C n^{1+\alpha-\kappa} n^{\frac{5 \alpha}{1-\alpha}}
$$

and the claim follows easily by the Borel-Cantelli lemma taking $\kappa$ large enough.

Next, we estimate the expected number of visits to $z \in \mathcal{D} \backslash\{y\}$ during an excursion that does not leave the disk. It is a well known fact that the expected number of visits of $z \in \mathbb{Z}^{2}$ by the simple random walk during one excursion from the origin is equal to one. So,

$$
\begin{aligned}
1= & \mathbb{E}[\# \text { visits of } z] \\
= & \mathbb{E}\left[\# \text { visits of } z \mid X_{d} \text { does not leave } \mathcal{D}\right] \mathbb{P}\left[X_{d} \text { does not leave } \mathcal{D}\right] \\
& +\mathbb{E}\left[\# \text { visits of } z \mid X_{d} \text { leaves } \mathcal{D}\right] \mathbb{P}\left[X_{d} \text { leaves } \mathcal{D}\right] .
\end{aligned}
$$


It follows that for $n$ large enough

$$
\begin{aligned}
& \mathbb{E}\left[\# \text { of visits of } z \mid X_{d} \text { does not leave } \mathcal{D}\right] \\
& \quad \leq(\mathbb{P}[\text { excursion does not leave } \mathcal{D}])^{-1} \\
& \quad \leq\left(1-G_{D\left(2^{n} n^{-\kappa}\right)}(0,0)^{-1}\right)^{-1} \leq 1+C / n \leq 2
\end{aligned}
$$

The expected duration of the $i$-th excursion, $V_{i}$, thus satisfies

$$
\mathbb{E}\left[V_{i}\right] \leq 2 \sum_{z \in \mathcal{D} \backslash\{y\}} \tau_{z} \leq 2 \sum_{z \in \mathbb{D}(n)} \tau_{z} \chi\left\{\tau_{z} \leq n^{-5 /(1-\alpha)} \varepsilon 2^{n / \alpha} / n\right\}
$$

The last sum can be bounded using Lemma 3.2. Let $i_{2}(n)$ be such that $2^{-i_{2}(n)} \leq$ $n^{-5 /(1-\alpha)} \leq 2^{-i_{2}(n)+1}$. Then, a.s. for $n$ large

$$
\begin{aligned}
\mathbb{E}\left[V_{i}\right] & \leq 2 \sum_{z \in \mathbb{D}} \tau_{z} \chi\left\{\tau_{z} \leq 2\right\}+2 \sum_{i=i_{2}(n)}^{i_{0}(n)} \sum_{z \in T_{\varepsilon 2^{-i}}^{\varepsilon^{-i+1}}} \tau_{z} \\
& \leq 4 \cdot 2^{n} n^{1-\alpha}+2 \sum_{i=i_{2}(n)}^{i_{0}(n)} \varepsilon \frac{2^{n / \alpha}}{n} 2^{-i+1}\left|T_{\varepsilon 2^{-i}}^{\varepsilon 2^{-i+1}}\right| \\
& \leq 4 \cdot 2^{n} n^{1-\alpha}+C 2^{n / \alpha} \sum_{i=i_{2}(n)}^{i_{0}(n)} 2^{-(1-\alpha) i} \leq C 2^{n / \alpha} n^{-5} .
\end{aligned}
$$

Since the expected number of excursions of $\bar{U}$ before time $(1+\theta) t$ is bounded by a multiple of $n$, the mean of the total time spent by $\bar{U}$ during the interval $\left[0,(1+\theta) t+2^{n / \alpha} / n^{2}\right]$ away from $y$ is easily bounded by $C 2^{n / \alpha} n^{-4}$ for $C$ depending on $\varepsilon$ but not on $t$.

We claim that (for $n$ sufficiently large) for any $u \in[0,(1+\theta) t], \mathbb{P}[\bar{U}(u) \neq$ $y] \leq 2 C / n^{2}$. Suppose not. Then for some $u_{0}, \mathbb{P}\left[Y\left(u_{0}\right) \neq y\right] \geq 2 C / n^{2}$. We have that the expected total time spent by $\bar{U}$ away from $y$ in interval $\left[u_{0}, u_{0}+2^{n / \alpha} / n^{2}\right]$ is bounded by $C 2^{n / \alpha} n^{-4}$, so there exists $v_{0} \in\left[u_{0}, u_{0}+2^{n / \alpha} / n^{2}\right]$ so that $\mathbb{P}\left[\bar{U}\left(v_{0}\right) \neq\right.$ $y] \leq C / n^{2}$. On the other hand, by the Markov property for $\bar{U}$ if $\lambda$ is the time of the first jump from $y$

$$
\begin{aligned}
\mathbb{P}\left[\bar{U}\left(v_{0}\right) \neq y\right] \geq \mathbb{P}\left[\bar{U}\left(v_{0}\right) \neq y \cap\left\{\lambda>v_{0}\right.\right. & \left.\left.-u_{0}\right\}\right] \\
& >\frac{1}{2} \mathbb{P}\left[\bar{U}\left(u_{0}\right) \neq y\right] \geq C / n^{2} .
\end{aligned}
$$

for $n$ sufficiently large. This contradiction gives the desired result and with it the lemma is proven. 


\section{Proof of subaging}

In this section we prove the subaging behaviour of the function $\Pi\left(t_{w}, t_{w}+t\right)$. Recall that this function has been defined as the probability that $X$ does not jump between $t_{w}$ and $t_{w}+t$. If we know that at time $t_{w}$ the process $X$ is in a trap $y$ with depth $\tau_{y}$, then this probability is easy to obtain, by the Markov property

$$
\mathbb{P}\left[X\left(t^{\prime}\right)=X(t) \forall t^{\prime} \in\left[t_{w}, t_{w}+t\right] \mid \tau_{X\left(t_{w}\right)}\right]=\exp \left(-\frac{t}{\tau_{X\left(t_{w}\right)}}\right) .
$$

We should thus gain an information about the depth $\tau_{X\left(t_{w}\right)}$. We would like to deduce its distribution from the behaviour of processes $\tilde{Y}^{n}$ and $\tilde{Y}$, because these are the only objects we really control. It should be obvious that the depth of the trap where $X$ is at time $t_{w}$ depends on the size of the jump of $\tilde{Y}^{n}$ that intersects the level $t_{w} / 2^{n / \alpha}$. Hence, to find an expression for the function $\Pi\left(t_{w}, t_{w}+t\right)$ we should control two basic objects. First, the distribution of the size of the jump of $\tilde{Y}^{n}$ that intersect certain level, and second, the conditional distribution of $\tau_{X\left(t_{w}\right)}$ knowing the size of this jump.

We start by controlling the size of the jump. Let $\ell_{n}=\ell_{n}(s)$ be the size of the jump of $\tilde{Y}^{n}$ that intersect the level $s$,

$$
\ell_{n}(s)=\inf \left\{x \in \mathcal{R}_{n}: x>s\right\}-\sup \left\{x \in \mathcal{R}_{n}: x \leq s\right\}
$$

and let $\ell=\ell(s)$ be the same size for the limiting process $\tilde{Y}$. We use $\mu_{s}^{n}$, resp. $\mu_{s}$ to denote the distributions of $\ell_{n}(s)$ and $\ell(s)$.

The following lemma is a consequence of Proposition 6.5 and the $\mathbb{P}$-a.s. continuity of the functional $Y \rightarrow \inf \{x \in \mathcal{R}(Y): x>s\}-\sup \{x \in \mathcal{R}(Y): x \leq s\}$ in the Skorokhod topology on $D([0, \infty))$.

Lemma 8.1. The sequence $\mu_{s}^{n}$ converges weakly to $\mu_{s}$ uniformly in $s \in\left[1,2^{1 / \alpha}\right]$, that is for every bounded continuous function $g$

$$
\int g(\ell) \mu_{s}^{n}(d \ell) \stackrel{n \rightarrow \infty}{\longrightarrow} \int g(\ell) \mu_{s}(d \ell) \quad \text { uniformly in } s \in\left[1,2^{1 / \alpha}\right] \text {. }
$$

As a consequence of the scaling invariance of $\tilde{Y}$ (recall that $\tilde{Y}$ is a stable subordinator) we get the following relation between the measures $\mu_{s}$,

$$
\mu_{s}([a, b])=\mu_{1}([a / s, b / s])
$$

for any interval $[a, b] \subset(0, \infty)$.

The control of $\tau_{X\left(t_{w}\right)}$ knowing the size of the jump is more complicated. It occupies the majority of the proof of the following proposition that is a refined version of Theorem 1.2.

Proposition 8.2. For $\mathbb{P}$-a.e. realisation of the environment $\boldsymbol{\tau}$,

$$
\lim _{t \rightarrow \infty} \Pi\left(t, t+\frac{\theta t}{\log t}\right)=\int_{0}^{\infty}\left(\frac{\ell \pi}{\ell \pi+\theta \alpha}\right)^{1+\alpha} \mu_{1}(d \ell) \equiv \Pi(\theta) .
$$


By an easy application of dominated convergence theorem we get

Corollary 8.3. The function $\Pi(\theta)$ satisfies

$$
\lim _{\theta \rightarrow 0} \Pi(\theta)=1 \quad \text { and } \quad \lim _{\theta \rightarrow \infty} \Pi(\theta)=0 .
$$

Proof of Proposition 8.2. We proceed similarly as in the proof of aging. We take $n(t)$ as in (152) and we define $s=s(t)=t / 2^{n(t) / \alpha}$. Next, we choose $\delta_{1}$ and $\delta_{2}$, and we set the constants $\varepsilon, M$ and $m$ in the same manner as before. We thus know that the process $\tilde{Y}^{n}$ is a good approximation of the rescaled time change $\bar{S}^{n}$. That means that $\mathbb{P}[A]=\mathbb{P}\left[A_{1} \cap A_{2}\right] \geq 1-C \delta_{1}$ with $A_{1}, A_{2}$ defined as in the previous section. For the following discussion we will suppose that $A$ occurs and we take account of the remaining part of the probability space at the end of the proof.

As we have already discussed, it is necessary to obtain the conditional distribution of $\tau_{X(t)}$ knowing $\ell_{n}(s)$. Similarly as in the proof of aging not much can be done if the distance between $s$ and $\mathcal{R}_{n}$ is smaller than $2 \delta_{2}$, because the approximation is not sufficiently precise. However, the probability of this bad case can be bounded by $C \delta_{1}+C^{\prime} \delta_{2}^{1-\alpha}$ uniformly in $s$ in the same way as in (171).

Let $E=E(n, s)$ denote the event $\operatorname{dist}\left(s, \mathcal{R}_{n}\right)>2 \delta_{2}$. If $E$ occurs, then the situation is more favourable. We know that $X$ was at time $t$ inside a disk $D_{y}\left(2^{n} n^{-\kappa}\right)$ around some deep trap $y=y(n, s)$. Moreover, similarly as in Lemma 7.4, we can show

$$
\mathbb{P}[X(t)=y(n, s) \mid E(n, s)] \rightarrow 1 \quad \text { as } \quad t \rightarrow \infty .
$$

We will thus compute the conditional distribution of $\tau_{y(n, s)}$ knowing $\ell_{n}(s)$ instead of the distribution of $\tau_{X(t)}$. As we have already discussed in the proof of Lemma 6.4, the size $\ell$ of the jump that is the result of the visit of $y$ satisfies

$$
2^{n / \alpha} \ell=\tau_{y} \sum_{i=1}^{\xi} e_{i}^{\prime},
$$

where $\xi$ is a geometrically distributed random variable with mean

$$
G_{D\left(2^{n} n^{-\kappa}\right)}(0,0)=n \log 2 / \pi+o(n)=\mathcal{K}^{\prime} n+o(n),
$$

and $e_{i}^{\prime}$ are i.i.d., exponential random variables with mean one. It is convenient to introduce the rescaled depth of trap, $\sigma_{x}=\tau_{x} n / 2^{n / \alpha}$. Equation (197) then becomes

$$
\ell=\frac{\sigma_{y}}{n} \sum_{i=1}^{\xi} e_{i}^{\prime} .
$$

As can be seen from Lemma 6.1, the distribution $v_{n}$ of $\sigma_{y}$ converges weakly to the distribution $v$ given by

$$
v(d x)=\frac{\alpha}{\varepsilon^{-\alpha}-M^{-\alpha}} \cdot \frac{1}{x^{\alpha+1}} d x \quad \text { for } \quad \varepsilon \leq x \leq M .
$$


The random variable $n^{-1} \sum_{i=1}^{\xi} e_{i}^{\prime}$ is an exponential random variable with mean $\mathcal{K}^{\prime}+o(1)$. Let $f_{n}$ denote its density, and let $f$ denote the density of the limiting distribution,

$$
f(x)=\exp \left(-x / \mathcal{K}^{\prime}\right) / \mathcal{K}^{\prime}
$$

We use $F_{\ell}^{n}$ to denote the distribution function of $\sigma_{y(n, s)}$ conditionally on $\ell_{n}(s)=\ell$,

$$
F_{\ell}^{n}(a)=\mathbb{P}\left[\sigma_{y(n, s)} \leq a \mid \ell_{n}(s)=\ell\right]
$$

Lemma 8.4. The function $F_{\ell}^{n}$ can be written as

$$
F_{\ell}^{n}(a)=\frac{\int_{\varepsilon}^{a} \frac{1}{x} f_{n}\left(\frac{\ell}{x}\right) v_{n}(d x)}{\int_{\varepsilon}^{M} \frac{1}{x} f_{n}\left(\frac{\ell}{x}\right) v_{n}(d x)}
$$

Proof. We should verify that for any event $B$ that is measurable with respect to the $\sigma$-algebra generated by the random variable $\ell_{n}(s)$

$$
\int_{B} \chi\left\{\sigma_{y} \leq a\right\} d \mathbb{P}=\int_{B} F_{\ell}^{n}(a) d \mathbb{P} .
$$

It is sufficient to verify the last expression for an event $B$ that has the form $\left\{\ell_{n}(s) \in\right.$ $I\}$ for some interval $I \subset[0, \infty)$. The left hand side of (204) can be then written as

$$
\int_{B} \chi\left\{\sigma_{y} \leq a\right\} d \mathbb{P}=\int_{\varepsilon}^{a} \int_{I / x} f_{n}(z) d z v_{n}(d x) .
$$

To compute the right hand side we should first find the distribution of $\ell_{n}(s)$

$$
\mathbb{P}\left[\ell_{n}(s) \leq u\right]=\int_{\varepsilon}^{M} \int_{0}^{u / x} f_{n}(z) d z v_{n}(d x) .
$$

The right hand side of (204) then equals

$$
\begin{array}{r}
\int_{I} \frac{\int_{\varepsilon}^{a} \frac{1}{x} f_{n}\left(\frac{\ell}{x}\right) v_{n}(d x)}{\int_{\varepsilon}^{M} \frac{1}{x} f_{n}\left(\frac{\ell}{x}\right) v_{n}(d x)} d\left(\int_{\varepsilon}^{M} \int_{0}^{\ell / x} f_{n}(z) d z v_{n}(d x)\right) \\
=\int_{I} \frac{\int_{\varepsilon}^{a} \frac{1}{x} f_{n}\left(\frac{\ell}{x}\right) v_{n}(d x)}{\int_{\varepsilon}^{M} \frac{1}{x} f_{n}\left(\frac{\ell}{x}\right) v_{n}(d x)}\left(\int_{\varepsilon}^{M} \frac{1}{x} f_{n}(\ell / x) v_{n}(d x)\right) d \ell
\end{array}
$$

Making the substitution $z=\ell / x$ and changing the order of integration it is easy to get the same expression as in (205). This finishes the proof.

As an consequence of the previous lemma we get 
Lemma 8.5. For any bounded continuous function $g$

$$
\int g(a) d F_{\ell}^{n}(a) \stackrel{n \rightarrow \infty}{\longrightarrow} \int g(a) d F_{\ell}(a),
$$

where

$$
F_{\ell}(a)=\frac{\int_{\varepsilon}^{a} z^{-\alpha-2} \exp \left(\ell / \mathcal{K}^{\prime} z\right) d z}{\int_{\varepsilon}^{M} z^{-\alpha-2} \exp \left(\ell / \mathcal{K}^{\prime} z\right) d z} .
$$

Moreover, if $K \subset(0, \infty)$ compact and $g$ has bounded first derivative, then the convergence is uniform in $\ell \in K$.

Proof. It is easy to show using the weak convergence of $v_{n}$ and properties of $f_{n}$ that the nominator, resp. the denominator of (203), converge to

$$
\int_{\varepsilon}^{b} \frac{1}{x} f\left(\frac{\ell}{x}\right) v(d x)
$$

with $b=a$ resp. $b=M$. Inserting (200) and (201) into the last expression we get

$$
\int_{\varepsilon}^{b} \frac{1}{x} f\left(\frac{\ell}{x}\right) v(d x)=\int_{\varepsilon}^{b} z^{-\alpha-2} \exp \left(\ell / \mathcal{K}^{\prime} z\right) d z,
$$

which proves the pointwise convergence. The uniform convergence can be then proved using standard methods.

We have now all ingredients to finish the proof of Proposition 8.2. Let $G=G(t)$ denote the event

$$
G=\left\{X\left(t^{\prime}\right)=X(t) \forall t^{\prime} \in[t, t+\theta t / \log t]\right\} .
$$

Then,

$$
\begin{aligned}
\mathbb{P}[G]= & \int_{0}^{\infty} \mathbb{P}\left[G \mid \ell_{n}(s)=\ell\right] \mu_{s}^{n}(d \ell) \\
= & \int_{0}^{\infty} \mathbb{P}[G \mid \ell \cap(A \cap E)]\left(1-\mathbb{P}\left[(A \cap E)^{c} \mid \ell\right]\right) \mu_{s}^{n}(d \ell) \\
& +\int_{0}^{\infty} \mathbb{P}\left[G \mid \ell \cap(A \cap E)^{c}\right] \mathbb{P}\left[(A \cap E)^{c} \mid \ell\right] \mu_{s}^{n}(d \ell)
\end{aligned}
$$

The second integral can be bounded by $\mathbb{P}\left[(A \cap E)^{c}\right] \leq C \delta_{1}+C^{\prime} \delta_{2}^{1-\alpha}$. The first one can be bounded from above by

$$
\int_{0}^{\infty} \mathbb{P}[G \mid \ell \cap(A \cap E)] \mu_{s}^{n}(d \ell) \equiv I(t)
$$

and from below by $I(t)-C \delta_{1}-C^{\prime} \delta_{2}^{1-\alpha}$. We should thus compute the value of $I(t)$. Using (190) we get

$$
I(t)=\int_{0}^{\infty} \int_{\varepsilon}^{M} \exp \left(-\frac{\theta t n}{a 2^{n / \alpha} \log t}\right) d F_{\ell}^{n}(a) \mu_{s}^{n}(d \ell) .
$$


Taking $t=s 2^{n / \alpha}$ we get

$$
I\left(s 2^{n / \alpha}\right)=\int_{0}^{\infty} \int_{\varepsilon}^{M} \exp \left(-\frac{\theta s \alpha}{a \log 2+c n^{-1} \log s}\right) d F_{\ell}^{n}(a) \mu_{s}^{n}(d \ell) . \quad \text { It is not }
$$

difficult to show using Lemmas 8.5 and 8.1, uniformly for $s \in\left[1,2^{1 / \alpha}\right]$, (216)

$\lim _{n \rightarrow \infty} I\left(s 2^{n / \alpha}\right)=\int_{0}^{\infty} \int_{\varepsilon}^{M} \exp \left(-\frac{\theta s \alpha}{a \log 2}\right) d F_{\ell}(a) \mu_{s}(d \ell) \equiv I_{\infty}(s) . \quad$ Inserting

(209) into (217) we get

$$
I_{\infty}(s)=\int_{0}^{\infty} \int_{\varepsilon}^{M} \exp \left(-\frac{\theta s \alpha}{a \log 2}\right) \frac{a^{-\alpha-2} \exp \left(-\ell / \mathcal{K}^{\prime} a\right)}{\int_{\varepsilon}^{M} z^{-\alpha-2} \exp \left(\ell / \mathcal{K}^{\prime} z\right) d z} d a \mu_{s}(d \ell) .
$$

For any $c>0$ the integral $\int_{0}^{\infty} \exp (-c / z) z^{-\alpha-2} d z=c^{-\alpha-1} \Gamma(\alpha+1)$. We introduce the following notation. Let

$$
g_{c}(\varepsilon, M)=\frac{1}{\Gamma(\alpha+1)}\left(\int_{0}^{\varepsilon}+\int_{M}^{\infty}\right) e^{-c / z} z^{-\alpha-2} d z
$$

and

$$
d_{1}=\frac{\theta s \alpha}{\log 2}+\frac{\ell}{\mathcal{K}^{\prime}} \quad \text { and } \quad d_{2}=\frac{\ell}{\mathcal{K}^{\prime}}
$$

Then

$$
I_{\infty}(s)=\int_{0}^{\infty} \frac{d_{1}^{-\alpha-1}-g_{d_{1}}(\varepsilon, M)}{d_{2}^{-\alpha-1}-g_{d_{2}}(\varepsilon, M)} \mu_{s}(d \ell) .
$$

The difference between $I_{\infty}(s)$ and $J(s) \equiv \int_{0}^{\infty}\left(d_{2} / d_{1}\right)^{1+\alpha} \mu_{s}(d \ell)$ is small for $\varepsilon$ small and $M$ large. To see this consider

$$
\begin{aligned}
& \lim _{\substack{\varepsilon \rightarrow 0 \\
M \rightarrow \infty}} I_{\infty}(s) \\
& \quad=\lim _{\substack{\varepsilon \rightarrow 0 \\
M \rightarrow \infty}}\left[\int \frac{d_{1}^{-\alpha-1}}{d_{2}^{-\alpha-1}-g_{d_{2}}(\varepsilon, M)} \mu_{s}(d \ell)-\int \frac{g_{d_{1}}(\varepsilon, M)}{d_{2}^{-\alpha-1}-g_{d_{2}}(\varepsilon, M)} \mu_{s}(d \ell)\right] .
\end{aligned}
$$

Both terms converge due to the monotone convergence theorem, first one to $J(s)$ and second one to 0 uniformly in $s$. From the scaling relation (193) we get that $J(s)$ actually does not depend on $s$,

$$
J(1)=\int_{0}^{\infty}\left(\frac{\ell \pi}{\ell \pi+\theta \alpha}\right)^{1+\alpha} \mu_{1}(d \ell) .
$$

Since $\varepsilon \rightarrow 0$ and $M \rightarrow \infty$ when $\delta_{1}, \delta_{2} \rightarrow 0$, there exists a function $h\left(\delta_{1}, \delta_{2}\right)$ such that $h\left(\delta_{1}, \delta_{2}\right) \rightarrow 0$ as $\delta_{1}, \delta_{2} \rightarrow 0$ satisfying $\left|I_{\infty}(s)-J(1)\right| \leq h\left(\delta_{1}, \delta_{2}\right)$ for all $s$. Using this, (217), (222), and the bounds in the paragraph after (213) we get that for $n$ larger than some $n\left(\delta_{1}, \delta_{2}\right)$ and for any $s \in\left[1,2^{1 / \alpha}\right]$

$$
\left|\mathbb{P}\left[G\left(s 2^{n / \alpha}\right)\right]-J(1)\right| \leq\left(C \delta_{1}+C^{\prime} \delta_{2}^{1-\alpha}+h\left(\delta_{1}, \delta_{2}\right)\right) .
$$

Since $\delta_{1}$ and $\delta_{2}$ can be taken arbitrarily small, the proof is finished. 


\section{Appendix A. Some properties of the simple random walk}

We summarise here some known properties of Green's function and hitting probabilities of the simple random walk on $\mathbb{Z}^{2}$ that is killed when it exits the disk $D$ with radius $r$. Let $\xi$ denote the exit time from this disk.

The most important formula that we use repeatedly is

$$
\mathbb{P}[X \text { hits } x \text { before } \xi]=1-\frac{\log |x|}{\log r}+O\left(\frac{|x|^{-2}}{\log r}\right)+O\left(\log ^{-2} r\right) .
$$

The proof of it can be found for example in Lawler [Law91], Proposition 1.6.7. We use also a similar expansion for the Green's function,

$$
G_{D}(0, x)=\frac{2}{\pi}(\log r-\log |x|)+O\left(|x|^{-2}\right)+O\left(r^{-1}\right) .
$$

For $G_{D}(0,0)$ there is the following formula ([Law91], Theorem 1.6.6)

$$
G_{D}(0,0)=\frac{2}{\pi} \log r+k+O\left(r^{-1}\right) .
$$

As an easy consequence of formula (225) we get following lemma:

Lemma A.1. Let y be an uniformly chosen point in D. Then there exists constant $C$ and $b<1$ independent of $r$ such that

$$
\mathbb{E}[\exp (b \log r \mathbb{P}[X \text { hits y before } \xi])] \leq C .
$$

Proof. Let $a$ be a positive constant and let $D_{a}$ denotes the disk with radius $a$. Then by (225) we have

$$
\begin{aligned}
& \mathbb{E}[\exp (b \log r \mathbb{P}[X \text { hits } y \text { before } \xi])] \\
& \quad \leq \frac{1}{\pi r^{2}} \sum_{y \in D_{a}} \exp (b \log r)+\frac{1}{\pi r^{2}} \sum_{D \backslash D_{a}} \exp (b \log r \mathbb{P}[X \text { hits } y \text { before } \xi]) \\
& \quad \leq \frac{C}{r^{2-b}}+\frac{1}{\pi r^{2}} \sum_{y \in D \backslash D_{a}} \exp \left\{b \log r-b \log |y|+O\left(|y|^{-2}\right)+O\left(\log ^{-1} r\right)\right\} \\
& \quad \leq C+\frac{1}{\pi r^{2-b}} \sum_{y \in D \backslash D_{a}} \frac{C}{|y|^{b}} \leq C+C r^{b-1} \int_{a}^{r} y^{-b} d y \leq C .
\end{aligned}
$$

This finishes the proof.

Similarly we get

Lemma A.2. There exist $\lambda>0$ and $C$ independent of $r$ such that

$$
\mathbb{E}\left[\exp \left(\lambda G_{D}(0, y)\right)\right] \leq C .
$$




\section{Appendix B. Some properties of stable subordinators}

Let $Y$ be a stable subordinator with the Lévy measure

$$
\pi(d x)=k x^{-\alpha-1} \chi\{x \geq 0\} d x, \quad k>0 .
$$

We use $\mathcal{R}=\mathcal{R}(Y)$ to denote the range of this process. Let $U(d x)$ denote its potential measure that is defined by

$$
U(A)=\int_{0}^{\infty} \mathbb{P}(Y(t) \in A) d t \quad \text { for any } A \in \mathcal{B}(\mathbb{R}) .
$$

For every $x>0$, let

$$
g(x)=\sup \{y \in \mathcal{R}: y \leq x\}
$$

and let

$$
d(x)=\inf \{y \in \mathcal{R}: y \geq x\} .
$$

Then it follows from Bertoin [Ber96], Theorems III.2, III.6, and the discussion following the second theorem that

Lemma B.1. (i) For each fixed $x \geq 0$ and every $0 \leq y \leq x<z$, we have

$$
\mathbb{P}(g(x) \in d y, d(x) \in d z)=U(d y) \pi(d z-y) .
$$

(ii) For every $x>0$ the random variable $x^{-1} g(x)$ has the distribution

$$
\frac{s^{\alpha-1}(1-s)^{-\alpha}}{\Gamma(\alpha) \Gamma(1-\alpha)} d s=\frac{\sin \alpha \pi}{\pi} s^{\alpha-1}(1-s)^{-\alpha} d s \quad(0<s<1) .
$$

\section{References}

[BBG03a] Ben Arous, G., Bovier, A., Gayrard, V.: Glauber dynamics of the random energy model. I. Metastable motion on the extreme states. Comm. Math. Phys. 235 (3), 379-425 (2003)

[BBG03b] Ben Arous, G., Bovier, A., Gayrard, V.: Glauber dynamics of the random energy model. II. Aging below the critical temperature. Comm. Math. Phys. 236 (1), 1-54 (2003)

[BČ04] Ben Arous, G., Černý, J.: Bouchaud's model exhibits two aging regimes in dimension one. To appear in Annals of Applied Probability (2004)

[Ben02] Ben Arous, G.: Aging and spin glass dynamics. Proceedings of Inter. Cong. Matematicians. Beijing 2002 III, 1-12 (2002)

[Ber96] Bertoin, J.: Lévy processes. Cambridge: Cambridge University Press, 1996

[Bi199] Billingsley, P. Convergence of probability measures. second ed., John Wiley \& Sons Inc., New York, 1999, A Wiley-Interscience Publication

[BM97] Bouchaud, J.-P., Mézard, M.: Universality classes for extreme-value statistics. J. Phys. A: Math. Gen. 30, 7997-8015 (1997)

[Bou92] Bouchaud, J.-P.: Weak ergodicity breaking and aging in disordered systems. J. Phys. I (France) 2, 1705-1713 (1992) 
[Čer03] Černý, J.: On two properties of strongly disordered systems, aging and critical path analysis. Ph.D. thesis, EPF Lausanne, 2003

[FIN02] Fontes, L.R.G., Isopi, M., Newman, C.M.: Random walks with strongly inhomogeneous rates and singular diffusions: convergence, localization and aging in one dimension. Ann. Probab. 30 (2), 579-604 (2002)

[Law91] Lawler, G.F.: Intersections of random walks. Birkhäuser Boston Inc., Boston, MA, 1991

[MB96] Monthus, C., Bouchaud, J.-P.: Models of traps and glass phenomenology. J. Phys. A 29, 3847-3869 (1996)

[RMB00] Rinn, B., Maass, P., Bouchaud, J.-P.: Multiple scaling regimes in simple aging models. Phys. Rev. Lett 84, 5403-5406 (2000) 